\title{
Inflammation induces pro-NETotic neutrophils via TNFR2 signaling
}

Friederike Neuenfeldt ${ }^{1,2^{*}}$, Jan Christoph Schumacher ${ }^{1,2^{*}}$, Ricardo Grieshaber-Bouyer ${ }^{3}$,

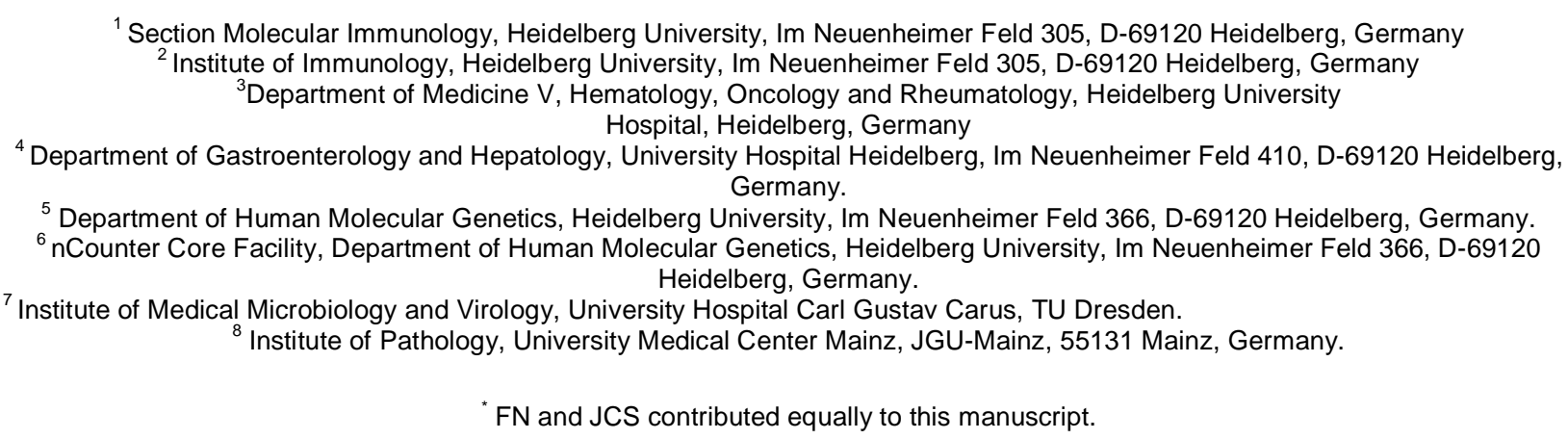




\section{Abstract}

29 Cytokines released during chronic inflammatory diseases induce pro-inflammatory 30 properties in polymorphonuclear neutrophils (PMN). Here we show that in vitro cytokine 31 treatment leads to the development of a subgroup of human PMN expressing CCR5, 32 termed $\mathrm{CCR}^{+}$cytokine-induced $\mathrm{PMN}\left(\mathrm{CCR} 5^{+} \mathrm{CPMN}\right)$. Auto/paracrine TNF signaling 33 increases intracellular neutrophil elastase (ELANE) abundance and induces NETosis in $34 \mathrm{CCR}^{+}$cPMN. Triggering of CCR5 amplifies NETosis. Membranous TNF (mTNF) 35 outside-in signaling induces the formation of reactive oxygen species, a known activator 36 of NETosis. In vivo, we find an increased number of $\mathrm{CCR} 5^{+} \mathrm{CPMN}$ in the peripheral 37 blood and inflamed lamina propria of patients with ulcerative colitis (UC) but not Crohn's 38 disease (CD). Notably, failure of anti-TNF therapy is associated with higher frequencies 39 of $\mathrm{CCR}^{+}{ }^{+} \mathrm{CPMN}$. In conclusion, we identify a phenotype of pro-NETotic, CCR5 positive

40 PMN present in inflamed tissue in vivo and inducible in vitro. These cells may reflect an 41 important component of tissue damage during chronic inflammation and could be of 42 diagnostic value. 


\section{Introduction}

44 Polymorphonuclear neutrophils (PMN) represent the first line of defense against

45 invading pathogens and are involved in inflammatory processes. PMN kill bacteria by

46 releasing anti-bacterial proteins (degranulation), production of reactive oxygen species

47 (ROS; oxidative burst) and by phagocytosis. Another important antibacterial mechanism

48 is the formation of neutrophil extracellular traps (NETs). NET formation - also termed

49 NETosis - is a process in which neutrophils discharge chromatin, forming an

50 extracellular mesh of DNA, oxidases, and proteases, e.g. neutrophil elastase (ELANE).

51 NETs are believed to capture and kill pathogens as well as to present them to other

52 phagocytes on a large scale (1). However, NETs can also initiate destructive processes

53 through e.g. interaction with other leukocytes. Consequently, exaggerated NETosis or a

54 failure in the removal of NETs by macrophages was shown to play an important role in

55 sustaining or exacerbating immune-mediated inflammatory diseases as well as cancer

$56(2-4)$.

57 Pro-inflammatory cytokines such as TNF occurring in inflamed tissues can induce

58 NETosis (5). On the other hand, the lifespan of PMN is strongly increased by pro-

59 inflammatory cytokines like IFN $\gamma$ or GM-CSF (6-9). Whether PMN die by NETosis or

60 survive by delaying apoptosis is an important decision point for progression of

61 inflammatory diseases. NETosis regulation has therefore moved into the focus of clinical

62 research. In that regard, one hypothesis claims that PMN subsets exist exhibiting

63 variable sensitivities to NETosis (10). Today it is known that PMN heterogeneity exists

64 due to circadian rhythm or homeostatic turnover (11-13) and functionally or

65 phenotypically altered PMN have been described in tumors, infections and in inflamed

66 tissues (14-19). It is currently unknown whether PMN diversification with an altered

67 functional repertoire or susceptibility to NETosis could explain increased NETosis in

68 inflammed tissues.

69 In this study, we analyzed functional and phenotypical alterations of human PMN under

70 inflammatory conditions. GM-CSF and IFN $\gamma$ were used to prime PMN, prolong their

71 survival and allow (trans)differentiation as described before for in vitro and in vivo

72 experiments (20-23). A gene expression analysis was performed with freshly isolated

73 human PMN and cytokine-stimulated PMN (cPMN). We discovered a cytokine-induced 
74 diversification of human PMN and significant upregulation of CCR5 on one cytokine-

75 depended subgroup, termed $\mathrm{CCR}^{+}$cytokine-depended (c)PMN.

\section{Results}

\section{Cytokine stimulation induces a CCR5-positive PMN subset}

79 We began by examining gene expression changes induced by extended cytokine

80 stimulation of human neutrophils. We purified resting whole blood PMN (rPMN) from

81 healthy volunteers by density centrifugation followed by magnetic bead isolation (Fig.

$821 \mathrm{~A}$ ), yielding 99\% pure CD66 ${ }^{+}$rPMN without eosinophil contamination (Supplementary

83 Figure 1A). rPMN were cultured with GM-CSF plus IFN $\gamma$ for 48 hours to obtain cytokine-

84 induced PMN (cPMN) as described previously (20-23). As expected, culture of PMN

85 without cytokines led to a higher rate of apoptosis and therefore further experiments were performed with cytokine-induced PMN (Supplementary Figure 1B).

We identified 106 differentially expressed genes between rPMN and cPMN at $\log _{2}$ fold change $\geq 2$ and a false discovery rate of 0.01. 38 genes were downregulated in cPMN and 68 genes were significantly up-regulated (Fig. 1B). CXCR2 was downregulated in stimulated PMN. Moreover, NLRP3, CASP8 and PYCARD, three transcripts related to the NLRP3 inflammasome-complex were downregulated. We also found downregulation of transcripts related to bacterial defense including CAMP (encoding cathelicidin) and CR1 (encoding complement receptor 1). Transcripts connected to apoptosis or PMN 94 removal (CASP8 and PECAM1), adhesion molecules (SELL and ITGB2) and 95 phagocytosis (ICAM3, PECAM1 and ITGB2) were also downregulated.

96 Among the upregulated transcripts were chemokines (CCL2, CCL3, CCL4, CCL8,

97 CCL13, CXCL2, CXCL9, CXCL10 and CCL24) and chemokine receptors (CCR1, 98 CCR5), the proinflammatory cytokine TNF, complement factors and complement 99 regulators $(C 1 Q B, C 3, S E R P I N G 1)$, a transcript encoding a catalytic subunit of the 100 NADPH-oxidase $(C Y B B)$ and transcripts encoding proteins involved in antigen101 presentation and lymphocyte regulation (HLA-DMA, HLA-DMB, HLA-DRA, HLA-DPA1, 102 HLA-DRB3, CD74, PSMB5, TAP1, TAP2, CD274, ICOSLG, CD48).

103 We then used the STRING database (Search Tool for the Retrieval of Interacting 104 Genes/Proteins) to analyze networks of predicted protein-protein interactions and 105 pathways among the differentially expressed genes (24). In this quality-controlled 
association database only direct (physical) interactions were included. Most up- and

107 downregulated genes were not connected. However, three closely connected clusters

108 comprising chemokine signaling via CCR1 and CCR5, antigen-processing, and NF-kB

109 signaling (including TNF) were among in the upregulated transcripts (Fig. 1C). Down-

110 regulated transcripts clustered in NLRP3 inflammasome-complex-related genes and

111 genes involved in adhesion and exocytosis.

112 Chemokine receptor expression was analyzed on protein level using flow cytometry. The

113 chemokine receptors CCR4 and CXCR3 were not expressed in rPMN and cPMN.

114 CXCR4 was expressed in low amounts on rPMN (CXCR4 ${ }^{\text {dim }}$ ) and was strongly

115 upregulated in CPMN (CXCR4 ${ }^{\text {bright }}$ ) (Fig. 1D). CCR1 was already expressed in rPMN, but

116 cytokine stimulation induced a bimodal expression pattern in CPMN. While CCR5 was

117 hardly detectable on the surface of rPMN, its expression was moderately upregulated on

118 cPMN. Notably, CCR5 was not upregulated on all cPMN, but again restricted to only a

119 subgroup of $\mathrm{CPMN}$. CCR $1^{\text {bright }}$ and CCR5-positive cPMN marked the same subgroup

120 (Fig. 1E). Thus, cytokine treatment induced two subgroups of cPMN characterized by

121 simultaneous expression or absence of CCR5, termed CCR5 ${ }^{+} \mathrm{cPMN}$ and CCR5 ${ }^{-} \mathrm{cPMN}$.

122 We next examined the frequency of PMN phenotypes across conditions. Less than $1 \%$

123 of whole blood (WB)-PMN or rPMN expressed CCR5. The amount of $\mathrm{CCR}^{+}$cells in

124 cPMN increased to $4-69 \%$ with a median of $17.4 \%$ after cytokine incubation (Fig. 1F).

125 Titration experiments covering a range of GM-CSF and IFN $\gamma$ observed in colitis models

126 in vivo showed that CCR5 expression was dose-dependent and increased in particular

127 with higher concentrations of INF $\gamma$ (Fig. 1G) (25-28). Interestingly, both INF $\gamma$ and GM-

128 CSF alone were sufficient to induce CCR5 ${ }^{+} \mathrm{cPMN}$, suggesting that this diversification

129 process is not unique to either cytokine. CCR5 was not detected 3h after adding of the

130 cytokines (day 0) and peaked after two days (Fig. 1H). Based on these experiments, 100

$131 \mathrm{U} / \mathrm{ml}$ GM-CSF + $10 \mathrm{ng} / \mathrm{ml} \mathrm{IFN} \gamma$ and an incubation time of 2 days were chosen as

132 optimum parameters.

\section{De novo expression and surface transport of CCR5}

135 Western blot analysis from whole cell lysates independently confirmed CCR5 expression

136 (Fig. 2A). Unexpectedly, CCR5 was expressed in both rPMN and cPMN at comparable

137 levels, suggesting intracellular storage of CCR5 and mobilization to the surface upon 
138 cytokine activation (Fig. 2A and B). In flow cytometry, freshly isolated PMN (rPMN) did

139 not show surface expression of CCR5, however, strong intracellular expression was

140 again recognizable in permeabilized cells (Fig. 2C). Similarly, cPMN contained high

141 levels of intracellular CCR5 but only a subgroup of CPMN expressed CCR5 on the cell

142 surface. Super-resolution microscopy using structured-illumination microscopy (3D-SIM)

143 revealed that CCR5 was stored in vesicular-like structures in rPMN and cPMN, while

144 surface localization was only detectable on a fraction of cPMN (Fig. 2D). A staining with

145 fluorescently-labelled phalloidin and imaging flow cytometry showed that the surface

146 expression of CCR5 was accompanied with lower F-actin content, while CRR5 ${ }^{\circ} \mathrm{cPMN}$

147 had an increased F-actin content (Fig. 2E). Accordingly, pertubation of actin dynamics

148 with cytochalasin D (CytoD) led to higher surface expression of CCR5 and stabilization

149 of F-actin with Jasplakinolide (Jas) diminished the amount of CCR5 on the cell surface

150 (Fig. 2F).

\section{Diversification-dependent regulation of surface receptors}

153 We next used flow cytometry to phenotype rPMN, CCR5 $5^{-}$and $\mathrm{CCR} 5^{+} \mathrm{cPMN}$ using a 154 selection of surface markers associated with neutrophil activation. Both $\mathrm{CCR}^{+}$and 155 CCR5 cPMN showed an activated phenotype with upregulation of CD66b (Fig. 2G), 156 while CD15 moderately upregulated on $\mathrm{CCR} 55^{-}$but not $\mathrm{CCR} 5^{+} \mathrm{CPMN}$. Further, CCR5 157 CPMN were $\mathrm{CD}_{2} \mathrm{~L}^{\text {dim }} \mathrm{CD} 16^{\text {bright }}$ and $\mathrm{CCR} 5^{+} \mathrm{CPMN}$ were $\mathrm{CD} 62 \mathrm{~L}^{\text {bright }} \mathrm{CD} 16^{\text {dim }}$. Notably, 158 the gene encoding CD62L (SELL) was also downregulated on mRNA level in activated 159 neutrophils (Fig. 1B).

160 The integrin CD49d ( $\alpha$-chain of the heterodimeric integrin VLA-4 (very-late-antigen-4) 161 was not expressed in CCR5 ${ }^{-}$and $\mathrm{CCR}^{+}{ }^{+} \mathrm{CPMN}$ (Fig. 2G). HLA-DR was not expressed 162 on $\mathrm{rPMN}$ but was induced on both $\mathrm{CCR} 5^{+}$and $\mathrm{CCR} 5{ }^{-} \mathrm{CPMN}$, mirroring the upregulation 163 of HLA-DRA on transcript level (Fig. 1C). The upregulation was stronger on CCR5 ${ }^{+}$ 164 cPMN (HLA-DR ${ }^{\text {bright }}$ ) compared to CCR5 cPMN (HLA-DR ${ }^{\text {dim }}$ ). CXCR1, one of the major 165 chemokine receptors expressed on PMN, was slightly downregulated on CCR5 and $166 \mathrm{CCR}^{+}{ }^{\mathrm{cPMN}}$ compared to rPMN (Fig. 2H). An upregulation of CXCR4 could be 167 observed on the surface of both CCR5 ${ }^{-}$and $C C R 5^{+} \mathrm{CPMN}$, with a higher expression on

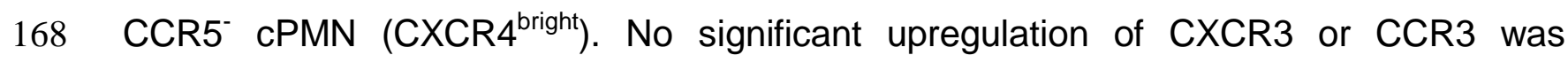
169 detectable on $\mathrm{CCR}^{-}$or $\mathrm{CCR}^{+}{ }^{\mathrm{CPMN}}$. Taken together, the cytokines GM-CSF and IFN $\gamma$ 
170 induce a diversification of PMN into CCR5 CXCR4 ${ }^{\text {bright } H L A-D R}{ }^{\text {dim }}$ CD62 ${ }^{\text {dim }}{ }^{\text {CD } 16}{ }^{\text {brigh }}$ and $\mathrm{CCR}^{+} \mathrm{CXCR} 4^{\text {dim }} \mathrm{HLA}-\mathrm{DR}{ }^{\text {bright }} \mathrm{CD} 62^{\text {bright }}{ }^{\text {CD } 16^{\text {dim }}} \mathrm{CPMN}$.

\section{CCR5 $^{+}$CPMN have a reduced migratory, exocytotic and phagocytic capacity}

174 The expression of CCR5 and CXCR4 on cPMN suggests a homing potential of these 175 cells towards inflamed sites. We used imaging flow cytometry to quantify the polarization

176 of PMN, which can be associated with migratory behavior (29). Cells were termed 177 polarized if they displayed formation of an F-actin rich protruding front (lamellipodium) 178 and a retracting rear, named uropod. cPMN were incubated with the CXCR4-ligand 179 SDF1 $\alpha(100 \mathrm{ng} / \mathrm{ml})$ or the CCR5-ligand CCL5 $(100 \mathrm{ng} / \mathrm{ml})$ for $5 \mathrm{~min}$, fixed and stained for

180 F-actin (phalloidin) and CCR5. While most cells had a round morphology in the absence 181 of chemokines, a clear polarization of F-actin and concomitantly a lamellipodium 182 formation could be observed for both CCR5 ${ }^{-}$and CCR5 ${ }^{+}$cPMN after SDF1 $\alpha$ stimulation 183 (Fig. 3A). In line with lower CXCR4 expression on $\mathrm{CCR}^{+} \mathrm{cPMN}$, their polarization in 184 response to SDF1 was weaker compared to CXCR4 ${ }^{\text {bright }} \mathrm{CCR}^{-} \mathrm{CPMN}^{\mathrm{Fig}}$. 3B). 185 Conversely, CCL5 induced a polarization in CCR5 ${ }^{+}$but not CCR5 ${ }^{-}$cPMN (Fig. 3A and 186 B). Thus, CCR5 ${ }^{-} \mathrm{CPMN}$ appear to be particularly responsive to CXCR4, while $\mathrm{CCR}^{+}$ cPMN are more sensitive to CCL5.

188 To test if polarization by SDF1 $\alpha$ or CCL5 also induces migration, we analyzed the 189 migratory behavior of CPMN in a transwell assay over three hours (Fig. 3C). Concordant 190 with polarization results from imaging flow cytometry, SDF1 $\alpha$, but not CCL5 induced 191 migration of $\mathrm{CXCR} 4{ }^{\text {bright }} \mathrm{CCR}^{-}$cPMN. Interestingly, we did not observe migration of $192 \mathrm{CXCR}^{\operatorname{dim}} \mathrm{CCR}^{+} \mathrm{CPMN}^{\mathrm{C}}$ in response to either CCL5 or SDF1 $\alpha$, suggesting that CCR5 ${ }^{+}$ 193 cPMN develop in inflammatory conditions, but do not migrate into inflamed sites on large 194 scale.

195 Next, we examined exocytosis and phagocytosis in rPMN and cPMN. Exocytosis was 196 assessed by flow cytometry analysis of surface expression of CD66b, which is stored in 197 the membrane of specific and tertiary granules and is transported to the cell surface 198 upon PMN activation. Both fMLP and ionomycin induced rapid exocytosis of CD66b in 199 rPMN starting after 10 min and reaching a maximum (>200\% of baseline) after 60 min. 200 (Fig. 3D). In contrast, cPMN showed a different exocytosis behavior. CD66b surface 201 expression was increased in both $\mathrm{CCR}^{-}$and $\mathrm{CCR}^{+}$cPMN only by ionomycin but not 
202 by fMLP. Moreover, the degree of ionomycin-induced CD66b upregulation was lower in

203 cPMN compared to rPMN. Importantly, the overall increase of CD66b on CCR5 ${ }^{+} \mathrm{cPMN}$ 204 was weaker compared to CCR5 cPMN.

205 To examine phagocytosis, we incubated rPMN or cPMN with GFP-expressing E. coli for 20615 minutes at $37^{\circ} \mathrm{C}$, which led to cytochalasin D-sensitive phagocytosis (Supplementary

207 Figure 2). Thereafter, cells were washed to remove free bacteria and analyzed using 208 flow cytometry (Fig. 3E). 28 $\pm 5 \%$ rPMN and 25 $\pm 3 \%$ of CCR5 cPMN, compared to $13 \pm 3 \%$ $209 \mathrm{CCR}^{+}$cPMN, phagocytosed at least one E. coli (Fig. 3F). Thus, cytokine-treatment per 210 se did not interfere with exocytosis and phagocytosis of PMN, but a strong and 211 significant reduction of these antibacterial properties could be observed for $\mathrm{CCR}^{+}$ 212 cPMN.

214 CCR5 $^{+}$CPMN are prone to spontaneous NET formation

215 Next, we analyzed the NETotic behavior of CCR5-defined populations of cPMN. CCR5 ${ }^{+}$ 216 cPMN were positively selected using magnetic beads, adhered on slides and NETosis 217 was analyzed by confocal microscope as described (Fig. 4A) (30, 31). NET formation 218 represents a multistep process including chromatin and nuclei reorganization, 219 accompanied with a rounding and swelling of the nuclei eventually leading to chromatin 220 expulsion (30). Thus, while the nuclei of mature PMN consist of 2-5 lobes, they de-lobe 221 during NETing and appear with a spherical shape which may result in de-nucleated 222 cytoplasts $(32,33)$ (Supplementary Figure 3A). In our experiments, CCR5 cPMN 223 showed mainly lobed nuclei (Fig. 4B). In contrast, CCR5 ${ }^{+}$cPMN showed a 224 heterogeneous shape of their nuclei, including lobed nuclei (39\% of the cells), round 225 nuclei (35\% of the cells), NET-shaped nuclei ( $8 \%$ of the cells) or denucleated cells $(18 \%$ 226 of the cells) which is related to NETosis $(32,33)$. Overview images confirmed ongoing 227 NETosis of $\mathrm{CCR}^{+}$cPMN (Supplementary Figure 3B) and, thus, these data clearly 228 indicate a pro-NETotic phenotype of $\mathrm{CCR}^{+} \mathrm{cPMN}$.

229 To rule out an effect of positive selection on NETosis, we also measured NETosis by 230 imaging flow cytometry without prior isolation. These analyses confirmed that rPMN and 231 CCR5 $^{-}$cPMN had mainly lobed nuclei, while lobed and round nuclei occurred in CCR5 ${ }^{+}$ 232 cPMN (Fig. 4C and D). In addition, imaging flow cytometry allows the calculation of the 233 chromatin swelling $(30,34)$. This analysis revealed that only a minor proportion of rPMN 
234 and CCR5 ${ }^{-}$CPMN (3\% and 8\%, respectively) had swollen nuclei (Fig. 4E, left and middle

235 dot plot), while a higher fraction of $\mathrm{CCR}^{+}$cPMN (19\%) showed this nuclear phenotype

236 indicating ongoing NETosis (Fig. 4E, right dot plot). For comparison, a known inducer of

237 NETosis was applied, i.e. the phorbol ester PMA, which increased the level of NETosis-

238 based nuclear morphology in rPMN significantly and of CCR5 ${ }^{-} \mathrm{CPMN}$ as well as CCR5 ${ }^{+}$

239 cPMN by trend. To substantiate the NETosis dynamics in CCR5 ${ }^{+}$cPMN, an end-stage

240 NETosis feature, i.e. the occurrence of denucleated cytoplasts, was evaluated (32, 33).

241 A small fraction of denucleated cytoplasts in rPMN (0.53\%) and CCR5 cPMN (2.3\%),

242 but a high number of $\mathrm{CCR}^{+}$cPMN cytoplasts (22.4\%) was observed (Fig. 4F).

243 Importantly, PMA-stimulation significantly increased the occurrence of $\mathrm{CCR}^{+} \mathrm{cPMN}$

244 cytoplasts.

245 Because CCL5 induced polarization of $\mathrm{CCR}^{+} \mathrm{cPMN}$ but not migration after 3h (see

246 above), it was reasonable to assume that CCR5-triggering activated signaling cascades

247 that led to enhanced NETosis. To investigate this, cPMN were incubated with CCL5 for

248 3h and NETosis was investigated by evaluating nuclear morphology. Indeed, NETosis

249 was again significantly increased by CCL5 in CCR5 ${ }^{+}$cPMN (Fig. 4G). This increase in

250 NETosis was independently attenuated by the MEK inhibitor U0126 and the reducing 251 agent/ROS antagonist N-acetyl-cysteine (NAC), but not by the calcium chelators BATA-

252 AM or EGTA, suggesting that ROS and MEK-dependent increased ROS levels are likely

253 to be responsible for CCL5-dependent increased NETosis (35). Indeed, CCL5 led to

254 ROS production in $\mathrm{CCR}^{+}$cPMN (Fig. 4H), which was strongest after 5 min and 255 deceased thereafter (Fig. 4I). Together, these results demonstrate that $\mathrm{CCR}^{+} \mathrm{cPMN}$ 256 are prone to spontaneous NET formation and that CCR5 triggering amplifies NETosis.

\section{Increased expression and nuclear localization of ELANE in $\mathrm{CCR5}^{\mathbf{+}} \mathbf{\text { cPMN }}$}

259 We next investigated why $\mathrm{CCR}^{+} \mathrm{cPMN}$ per se exhibited a pro-NETotic phenotype.

260 NETosis is a highly regulated process in which signaling cascades induce relocation of

261 ELANE from azurophilic granules to the nuclei, where it participates in NETosis-related

262 chromatin decondensation. Intracellular flow cytometry revealed a significant increase in

263 ELANE expression in $\mathrm{CCR}^{+}$cPMN compared to both CCR5 ${ }^{-}$cPMN and rPMN (Fig. 5A 264 and B). Imaging flow cytometry disclosed that ELANE was mainly localized in the 265 cytoplasm in CCR5 cPMN (Fig. 5C, upper panel). In contrast, ELANE displayed a 
266 nuclear localization in $\mathrm{CCR}^{+}$cPMN (Fig. 5C, lower panel, yellow color). A rescaled ELANE/DAPI Pearson correlation coefficient confirmed that ELANE was mainly in the cytoplasm of CCR5 ${ }^{-}$cPMN, and a nuclear translocation of ELANE was observed in $38.7 \pm 4.3 \%$ of $\mathrm{CCR}^{+}$cPMN (Fig. 5D and E). In conclusion, an increased expression and preferentially nuclear distribution of ELANE provide a mechanistic basis for the increased NETosis in CCR5 ${ }^{+}$cPMN.

273 Dichotomic function of soluble (sTNF) and membrane bound TNF (mTNF) on 274 NETosis of CCR5 $^{+}$cPMN

275 Since stimulation of PMN with GM-CSF plus IFN $\gamma$ induced transcription of TNF, we speculated that IFN $\gamma$ could induce TNF expression in CPMN which then could regulate ELANE expression and NETosis in $\mathrm{CCR}^{+} \mathrm{cPMN}$ in an para- or autocrine manner. By

278 intracellular flow cytometry, TNF expression could already be detected at low levels in 279 rPMN but increased in cPMN, especially in $\mathrm{CCR}^{+} \mathrm{cPMN}$ (Fig. 6A, left graph). The 280 Tumor necrosis factor receptor 1 (TNFR1) was highly expressed on CCR5 cPMN and 281 dimly on $\mathrm{CCR}^{+}$cPMN or rPMN. Tumor necrosis factor receptor 2 (TNFR2) showed the opposite pattern, with high expression on $\mathrm{CCR}^{+}$but not CCR5 ${ }^{-}$cPMN (Fig. 6A, middle 283 and right graph).

284 We next analyzed signaling pathways downstream of TNF. PI3Kinase inhibition but not 285 IkB kinase $\beta$ inhibition interfered with ELANE upregulation in $\mathrm{CCR}^{+} \mathrm{cPMN}$ (Fig. 6B). 286 Notably, inhibition of PMN-secreted TNF by a blocking antibody also led to decreased 287 ELANE expression in $\mathrm{CCR}^{+}$cPMN (Fig. 6B). Accordingly, application of anti-TNF or 288 PI3Kinase inhibition during the diversification process reduced NETosis only in CCR5 ${ }^{+}$ 289 cPMN, but not in CCR5 cPMN (Fig. 6C). In contrast, NF-kB inhibition reduced 290 spontaneous NETosis in both CCR5 ${ }^{-}$cPMN and CCR5 ${ }^{+}$cPMN, suggesting that a 291 process downstream of TNF is involved in ELANE expression. Indeed, stimulation of

292 rPMN with recombinant human TNF (rhTNF) was sufficient to induce ELANE 293 expression (Fig. 6D).

294 Since the two TNF receptors differ in their ability to recognize soluble versus membrane 295 bound TNF, we examined the distribution of TNF in cPMN. Imaging flow cytometry 296 revealed that the majority of cellular TNF was membrane-bound on $\mathrm{CCR}^{+} \mathrm{cPMN}$, while 297 it resided intracellularly in $\mathrm{CCR5}^{-}$cPMN (Fig. 6E). mTNF $\alpha$ abundance was significantly 
298 higher on $\mathrm{CCR}^{+}$cPMN when compared to CCR5 ${ }^{-}$cPMN, suggesting that TNF-mediated

299 outside-in signaling may be possible in CCR5 ${ }^{+}$cPMN (Fig. 6F).

300 To test this hypothesis, we applied anti-TNF antibodies to already diversified CCR5 ${ }^{+}$

301 cPMN expressing mTNF and TNFR2. Flow cytometry revealed that cPMN contained

302 higher ROS levels compared to rPMN. Anti-TNF treatment induced an additional

303 increase in intracellular ROS, and a similar increase was found after addition of the

304 CCR5 ligand CCL5 (Fig. 6G). Concordantly, NETosis was increased by anti-TNF

305 treatment of cPMN (Fig. 6H). Thus, anti-TNF decreases ELANE expression and

306 spontaneous NETosis when applied during the diversification of PMN but increases

307 ROS-dependent NETosis when cPMN are already diversified (compare Model in Fig. 8)

308 (36).

310 CCR5 $^{+}$PMN are abundant in the mucosa of patients with ulcerative colitis

311 PMN are associated with intestinal inflammation in inflammatory bowel disease, a

312 disease group in which TNF blockade is often used successfully for treatment $(37,38)$.

313 To determine whether increased CCR5 expression on PMN is detectable in IBD

314 patients, sections of inflamed colonic tissue specimens of UC and CD patients and

315 healthy controls ( $\mathrm{HC})$ were analyzed by immunohistochemistry. Azurocidin was used to

316 visualize PMN (Fig. 7A, green). As expected, the frequency of PMN was higher in the

317 lamina propria of CD and UC patients compared to controls. Notably, CCR5 colocalized

318 with azurocidin and CCR5 expression on PMN could only be observed in the lamina

319 propria of UC patients, but not in the colon of CD patients or healthy controls (Fig. 7A,

320 red, quantification in Fig. 7B). In crypt abscesses, which are histologically defined by

321 PMN exudates, the frequency of CCR5 expressing PMN was again significantly

322 increased.

323 We utilized the loss-of-epithelial-layer (LEL) system (39) to analyze whether the

324 intestinal micro-millieu can induce diversification of human PMNs. In this explant model,

325 the epithelial layer is detached from surgically obtained colon preparations by EDTA

326 treatment, resulting in tissue inflammation and secretion of cytokines into the

327 supernatant. We incubated human PMN from healthy donors with LEL supernatant and

328 subsequently quantified the fraction of $\mathrm{CCR}^{+} \mathrm{cPMN}$ (Fig. 7C). These experiments 
329 demonstrated that LEL supernatant diversifies PMNs to $\mathrm{CCR}^{+}{ }^{\mathrm{cPMN}}$, suggesting that

$330 \mathrm{CCR5}^{+}$cPMNs can develop locally in inflamed tissues.

331 We further examined whether $\mathrm{CCR}^{+}$PMN could also be detected with surface

332 staining of leukocytes in the peripheral blood of IBD patients. For this purpose, blood

333 samples were collected from healthy controls and patients with UC and CD and flow

334 cytometric analysis was performed without prior PMN isolation (Fig. 7D). Indeed, a

335 significantly higher proportion of $\mathrm{CCR}^{+} \mathrm{CPMN}$ was found in UC patients compared to

$336 \mathrm{HC}$ and $\mathrm{CD}$. The increased abundance of $\mathrm{CCR}^{+}$PMN did not correlate with CRP

337 level, disease score, or fecal calprotectin (Fig. 7E). However, we found a significant

338 increase in the proportion of $\mathrm{CCR}^{+} \mathrm{CPMN}$ in UC patients in whom anti-TNF therapy

339 (infliximab or adalimumab) failed (Fig. 7F).

340 We conclude that soluble factors released from inflamed intestine in vitro induce a

341 phenotype of $\mathrm{CCR}^{+} \mathrm{PMN}$ which can also be found in inflamed mucosa in vivo and are

342 detectable at increased frequency in the blood of patients who failed anti-TNF

343 therapy. 


\section{Discussion}

346 PMN are not only bactericidal cells, but play a very important role in maintaining the

347 inflammatory background of chronic inflammation. By producing extracellular traps and 348 secreting of cytokines, PMN contribute to smoldering of inflammation and destruction of

349 inflamed tissues. This function of PMN can be accompanied by PMN re-programming, 350 i.e. a change in their phenotype and functionality. Here, we show that PMN can diversify 351 into two distinct subgroups, i.e. CCR5 $^{-}$(TNFR1 ${ }^{\text {bright }} \mathrm{CXCR} 4^{\text {bright } H L A-}$ $\mathrm{DR}^{\operatorname{dim}}$ CD62L $\left.{ }^{\operatorname{dim}} \mathrm{CD} 16^{\text {brigh }}\right)$ and $\mathrm{CCR}^{+}$ (TNFR2 ${ }^{\text {bright }}$ CXCR4 ${ }^{\text {dim } H L A-~}$

$353 \mathrm{DR}^{\text {bright }} \mathrm{CD} 62 \mathrm{~L}^{\text {bright }} \mathrm{CD} 16^{\text {dim }}$ ) $\mathrm{CPMN}$. The latter subgroup showed decreased anti-bacterial 354 armamentarium with the exception of NETosis, which was accelerated. $\mathrm{CCR}^{+} \mathrm{cPMN}$ 355 were found in inflamed colon of UC patients. There, a NETosis-related release of

356 ELANE might be an important trigger for the inflammation and tissue destruction in the 357 gut of UC patients.

358 Expression of CCR5 on PMN is also evident in other contexts. A subgroup of PMN 359 derived suppressor cells expressing CCR5 was described in mice (40). In this model, 360 inhibiting CCR5 interfered with chemoattracting these cells into tumors and, thereby, 361 interfered with their immuno-suppressive function. CCR5 expression on apoptotic PMN 362 had anti-inflammatory functions by locally sequestering CCR5 ligands, a mechanism 363 shown to take part in resolution of experimental peritonitis (41). This study supports our 364 hypothesis that CCR5 transport to the cell surface represents a proximal priming event 365 (Fig. 8A). Cytokine-mediated stimuli (e.g. GM-CSF), however, prevent apoptosis and 366 prolong survival (42) as also evident by downregulation of CASP8 gene in our study.

367 Additional gene expression and TNF upregulation can be provoked by the IFN $\gamma /$ IFNR 368 axis modulating PMN functionality. Thus, the sequence of events acting on PMN during 369 the course of inflammation determine the fate of individual PMN.

370 Therefore, based on our data we propose the following model: PMN priming induces 371 immediate transport of granule-stored CCR5 to the cell surface (Fig. 8A). A high 372 deviation in the $\mathrm{CCR}^{+}$cPMN frequency occurred if PMN of the same donor were re373 analyzed in otherwise independent experiments (data not shown) indicating that the 374 observed diversification and development of $\mathrm{CCR}^{+} \mathrm{CPMN}$ is independent on epigenic 375 mechanisms. Rather, this process is coupled to actin dynamics since perturbation of the 376 actin cytoskeleton by CytoD increased CCR5 surface expression (43-45). At this stage, 
377 PMN are prone to apoptosis unless they receive survival signals. In the presence of

378 cytokines, apoptosis is delayed, de novo synthesis reinforces CCR5 expression and a 379 three hit sequence is initated in $\mathrm{CCR}^{+}$cPMN (Fig. 8B). In a first hit, IFN $\gamma$ induces the 380 expression of TNF. In the second hit, TNF binds to the TNFR2 leading to PI3K381 dependent upregulation of ELANE expression and rendering (together with the 382 disassembly of the actin cytoskeleton (46)) $\mathrm{CCR}^{+}$cPMN pro-NETotic. The third hit is 383 mediated by increasing intracellular ROS levels. The amplification is due to ROS384 dependent activation of ELANE (47) and is provoked by triggering of CCR5 dissociating 385 CCR5 proximal signaling from CCR5-induced migration. This is in line with the reverting 386 effect of ROS-scavenger NAC and with the upregulation of CYBB gene in cPMN.

387 According to this three hit model, TNF plays a role for the development of $\mathrm{CCR}^{+} \mathrm{cPMN}^{\mathrm{C}}$ 388 as well as ELANE upregulation. It is known that the soluble moiety of TNF (sTNF) is 389 released after proteolytic cleavage of the transmembrane isoform (mTNF) by TNF390 converting enzyme (TACE; ADAM-17) (48). While TNFR1 binds soluble TNF, TNFR2391 mediated signaling is also induced by sTNF and mTNF. Notably, soluble and membrane 392 bound TNF can exert opposing biological effects and TNF directed antibodies can 393 induce outside-in signaling or mTNF/TNFR2 crosslinking (36, 49, 50). The 394 therapeutically used TNFR2-fusion protein (eternacept) does not bind to mTNF and can, 395 thus, not induce outside-in-signaling. It was demonstrated that eternacept was not 396 beneficial for treatment of IBD patients and patients with other autoimmune diseases 397 and treated with eternacept had an increased risk of being diagnosed with IBD $(51,52)$.

398 One possible explanation of this paradoxical effect is that eternacept shifts the balance 399 of sTNF/TNFR1- toward mTNF/ TNFR2-signaling promoting the pro-NETotic state of $400 \mathrm{CCR}^{+}$cPMN. According to our model, anti-TNF antibodies can exert different biological 401 effects on PMN, i.e. inhibiting ELANE expression and NETosis (Figure 8C) or induction 402 of ROS and consequently accelerate NETosis (Figure 8D). Thus, besides the well 403 described effects of anti-TNF therapy (53-56), its net result could be modulated by the 404 PMN diversification state. This would explain why patients with higher $\mathrm{CCR}^{+} \mathrm{cPMN}$ 405 frequency tend to be more prone to failure of anti-TNF therapy. Further prospective 406 studies in colitis patients are needed to test this hypothesis.

407 Although there are a plethory of evidences showing that CCR5 is involved in chronic 408 inflammatory diseases (reviewed in (57)) the current view on CCR5 in IBD is ambiguous. 
409 On the one hand, there is no gene polymorphism of CCR5 is associated with IBD (58, 410 59). On the other hand, the CCR5-ligands CCL3 (MIP-1 $\alpha$ ), CCL4 (MIP-1 $)$ ), CCL5

411 (RANTES) and CCL8 (MCP-2) are expressed in the colon of IBD patients (60) and 412 blockage of CCR5 ameliorated DSS/TNBS-induced colitis in mice models (61). In

413 addition, dominant inactive CCL5 reduced tissue damage in experimental rat models

414 (62). One reason for such ambiguity is that the individual frequency of $\mathrm{CCR}^{+} \mathrm{cPMN}$ in

415 patients was not considered in previous studies and CCR5 detection in tissues has

416 certain drawbacks: A plethora of spatial distributed CCR5 conformations and CCR5

417 oligomerization exsist $(63,64)$ and CCR5-specific antibodies display varying 418 conformation-dependent affinities (65). This might also explain why PMN lacking CCR5

419 expression were observed in our gut tissue samples; antigen retrieval of FFPE420 embedded tissue samples might lead to conformation-restricted epitope liberation.

421 Nevertheless, infiltration of PMN into colonic mucosa and NETosis is considered a 422 hallmark of UC (66-70), and PMN activity is substantially enhanced in the colon of active 423 UC patients (71). Very little is known about the occurrence and function of PMN 424 subgroups. The here described $\mathrm{CCR}^{+} \mathrm{CPMN}$ have pro-inflammatory properties. By 425 producing TNF and an increased tendency to undergo NETosis, these cells likely 426 contribute to disease deterioration rather than improvement. Since NET associated 427 ELANE was shown to have higher proteolytic activity on the extracellular matrix 428 compared to unbound ELANE (72), the NETosis-associated release of ELANE could be 429 an important step for exacerbating colitis. In line with this assumption, fecal ELANE is a 430 predictive clinical marker for activity of UC in patients (73).

431 PMN heterogeneity represents a still underestimated factor influencing the pathogenesis 432 of inflammatory diseases. It can have different sources, and whether distinct subsets of 433 PMN exist or if they represent different states along a continuous distribution is an area 434 of active research $(12,74,75)$. It was recently shown that PMN can be represented 435 along a continuous, chronologically ordered spectrum, termed neutrotime, from which 436 they can deviate to reach different polarization states as a function of tissue, stimulus 437 and timepoint (76). Since inflamed PMN were mostly advanced along neutrotime, $438 \mathrm{CCR}^{+} \mathrm{CPMN}$ might develop from cells in late neutrotime. Deviation from neutrotime can 439 occur at different stages, and the conditions in the immediate mirco-milleu as well as the 440 sequence of stimuli determine PMN plasticity in inflammation. Thus, different flavours of 
$441 \mathrm{CCR}^{+} \mathrm{cPMN}$ might exsist and whether the here described $\mathrm{CCR}^{+} \mathrm{cPMN}$ and $\mathrm{CCR}^{-}$ 442 cPMN are temporally related activation states or divergent, independent subgroups 443 originating from a common sequence and their relationship to other PMN described 444 subgroups (77) remains to be determined.

445 Taken together, the here described biological properties of $\mathrm{CCR}^{+} \mathrm{cPMN}$ and their 446 occurrence in UC-dependent cryptitis could represent another important piece of the 447 puzzle for understanding UC pathogenesis and emphazises the need of standardized 448 PMN phenotyping in inflammatory diseases. 


\section{Material and Methods}

\section{Antibodies and Reagents}

453 The following primary antibodies were employed: CCR5-PE (Miltenyi, Bergisch 454 Gladbach, Germany); CCR5 unlabeled; CCR1-APC-Fire 750, CXCR1-APC, CCR4455 BV605, CD66b-PerCP-Cy5.5, CD15-AF594, CD62L-FITC, CD49d-APC, CD16-APC456 Cy7, HLA-DR-AF700, TNF $\alpha$-PE-Dazzle594, TNFR1-APC, TNFR2-PE-Cy7 (Biolegend, 457 San Diego, USA); CXCR3-APC, CXCR3-PE-Cy5, CXCR4-PE-Cy5, CCR3-PE CCR3458 PE-CF594, CD15-BV605, CD49d-PE-Cy5 (BD Bioscience, Heidelberg, Germany); 459 ELANE (MerckMillipore, Darmstadt, Germany); CXCR4-FITC (R\&D Systems, 460 Minneapolis, USA); TNF $\alpha$ (purified), CCR5 (purified) (R\&D Systems, Minneapolis, USA); azurocidin (Abcam, Cambridge, UK); Anti-mouse-Cy5 (Dianova, Hamburg, Germany).

462 DAPI, Phalloidin, ionomycin, paraformaldehyde, PMA and CytoD were from 463 MerckMillipore (Darmstadt, Germany), TPCA from Abcam (Cambridge, UK), GM-CSF 464 and CCL5 from R\&D Systems (Minneapolis, USA), IFN $\gamma$ from Biolegend (San Diego, 465 USA).

\section{Isolation of human peripheral blood neutrophils (PMN)}

468 PMN were purified from 30-90ml peripheral blood of healthy donors. The isolation of 469 PMN was performed through density centrifugation. Briefly, for each preparation 30ml of 470 whole blood was added to $20 \mathrm{ml}$ of PolymorphPrep ${ }^{\mathrm{TM}}$ and centrifuged at $535 \mathrm{~g}$ for $35 \mathrm{~min}$.

471 The PMN layer was collected, washed with $0.45 \%$ sodium chloride solution in a $1: 1$ ratio 472 and centrifuged at $443 \mathrm{~g}$ for $10 \mathrm{~min}$. To remove erythrocyte contamination, the pellet was 473 mixed with $20 \mathrm{ml}$ of $0.2 \%$ sodium chloride solution. The resulting hemolysis then was 474 stopped after 10 seconds through adding $20 \mathrm{ml}$ of $1.6 \%$ sodium chloride solution. After 475 washing twice, the pellet was resuspended in RPMI +10\% FCS. For mRNA analysis, a 476 second bead-based purification step was included according to the manufacturer's 477 instructions (EasySep ${ }^{\mathrm{TM}}$ Human Isolation Kit from STEMCell Technologies, Cologne, 478 Germany). While density centrifugation led to $90 \%$ pure PMN, the two-step isolation 479 protocol allowed to reach 99.7\% PMN purity. After purification, cells were seeded with a 480 mixture of GM-CSF (100 U/ml) plus IFN $\gamma(10 \mathrm{ng} / \mathrm{ml})$ in 6-well-plates $(5 \mathrm{ml}$ per well) and 481 incubated at $37^{\circ} \mathrm{C}$ and $5 \% \mathrm{CO}_{2}$ for 48 hours. 
482 For bead-based isolation of human $\mathrm{CCR}^{+}$and CCR5 ${ }^{-}$cPMN were sorted using anti483 APC MojoSort ${ }^{\mathrm{TM}}$ nanobeads (Biolegend, San Diego, USA) according to the 484 manufacturer's recommendations. Briefly, PMN were stimulated with GM-CSF (100 $485 \mathrm{U} / \mathrm{ml})$ plus IFN $\gamma(10 \mathrm{ng} / \mathrm{ml})$ for one day. $2 \times 10^{6}$ cells were collected and labeled with APC486 labelled CCR5 antibodies (Miltenyi, Bergisch Gladbach, Germany) in RPMI 487 supplemented with 10\% FSC, 2 mM EDTA and $10 \mathrm{mM}$ HEPES for $20 \mathrm{~min}$. After washing $48812.5 \mu$ anti-APC MojoSort ${ }^{\mathrm{TM}}$ nanobeads and $1 \mathrm{ml}$ RPMI supplemented with 10\% FSC, 2 $489 \mathrm{mM}$ EDTA and $10 \mathrm{mM}$ HEPES were added. After $20 \mathrm{~min}$. incubation time, cells were 490 isolated using a magnet.

\section{Flow cytometry}

493 PMN were fixed with 1.5\% PFA for 10 min followed by one washing step with 494 FACSwash buffer (PBS, 10g/l BSA, 0.1\% $\mathrm{NaN}_{3}$ - for intracellular staining $0.1 \%$ saponin 495 was added). All washing steps were completed by centrifugation at $300 \mathrm{~g}$ for $6 \mathrm{~min}$. The 496 PMN pellet was then incubated light-protected with $50 \mu \mathrm{l}$ of FACSwash-antibody-mix for 49720 minutes. Thereafter, excess antibodies were removed by washing with FACSwash 498 buffer. For whole blood staining, $200 \mu$ of heparinized whole blood were incubated with $49950 \mu \mathrm{l}$ antibody-mix for $30 \mathrm{~min}$ protected from light at $4^{\circ} \mathrm{C}$. Red blood cells were removed 500 by adding $2 \mathrm{ml}$ of BD FACS Lysing Solution (BD Bioscience, Heidelberg, Germany) for $50110 \mathrm{~min}$ at room temperature. The cells were then washed twice with FACSwash buffer 502 (PBS, 10g/l BSA, 0.1\% $\mathrm{NaN}_{3}, 0.1 \%$ saponin). Cells were measured using an LSRII flow 503 cytometer (BD Bioscience, Heidelberg, Germany) and analyzed using FACSDiva ${ }^{\mathrm{TM}}$ 504 software version 8 and FlowJoTM version 10.6.0 (BD Bioscience, Heidelberg, Germany).

505 To identify PMN, cells were gated according to the FSC/SSC profile combined with

506 CD66b expression.

508 Imaging flow cytometry

509 For imaging flow cytometry, samples were prepared as described for conventional flow

510 cytometry (see above), and cells were finally resuspended in 50 $\mu$ PBS/ 5\%BSA.

511 Thereafter, 25,000 cells were acquired using an ImageStream IsX Mkll and Inspire 512 software (Amnis, Seattle, USA). Data analysis was performed with IDEAS 6.2.188.0 513 (Amnis, Seattle, USA) (78, 79). Briefly, cells were gated according to the cell size (area 
514 brightfield) and side scatter signal as well as their CCR5-expression. Cell polarization

515 was quantified according to the aspect ratio $(A R)$ of the phalloidin signal. Polarized cells 516 were considered as such, if the AR was below 0.85. For assessing the nuclear 517 morphology on the single cell level, an DAPI-dependent threshold mask was applied 518 (M07, Ch07, 40; (for details about masking see $(78,80,81)$ )), and the distances of the 519 mask center to the boundaries were measured (circularity feature). Higher circularity 520 values indicated rounder shapes of the nuclei, whereas low values indicated lobular 521 nuclei. During NETosis the nuclear membrane dissolves and the chromatin begins to 522 swell. Microscopically, chromatin swelling can be determined by a decrease in the 523 contrast of nuclear images combined with an increase of the area covered by the 524 chromatin (34). To quantify these morphological characteristics of the chromatin, we 525 calculated the root mean square for image sharpness, i.e. the "Gradient RMS" feature, 526 which correlates with the contrast of the nuclear images and was calculated by imaging 527 flow cytometry and IDEAS software. In addition, the area of the nuclei in metric numbers $528\left(\mu \mathrm{m}^{2}\right)$ was calculated.

\section{Phagocytosis assay}

531 Ampicillin-resistant, GFP-expressing Escherichia coli were grown overnight in lysogeny 532 broth (LB medium) at $37^{\circ} \mathrm{C}$ with continuous rotation at $230 \mathrm{rpm}$. After 12 hours, the 533 number of bacteria was assessed using turbidity measurement and adjusted to $10^{7}$ $534 \mathrm{bacteria} / \mathrm{ml}$ in RPMI + 10\% FCS. PMN were prepared in aliquots of $1 \mathrm{ml}(\mathrm{RPMl}+10 \%$ 535 FCS) containing $1 \times 10^{6}$ cells. To each PMN sample, $50 \mu \mathrm{l}$. coli were added to achieve 536 a PMN-bacteria-ratio of $1: 10$ or $1: 12$, respectively. The samples were incubated at $37^{\circ} \mathrm{C}$ 537 for 20 min and washed once with RPMI + 10\% FCS and once with PBS. Cells were fixed 538 and stained with fluorochrome labelled antibodies as indicated and measured 539 immediately with conventional or imaging flow cytometry.

541 Loss-of-epithelial layer model

542 Mucosal specimens were washed extensively in RPMI 1640 (Life Technologies, Paisley, 543 UK) containing antibiotics prior to removing the mucus layer by dithiothreitol (DTT) 544 treatment (1 mM, 10 min; Sigma, St. Louis, MO, Germany). Subsequently, punches of 545 defined surface area were prepared and denuded of epithelial cells by exposure to 0.7 
546 mM EDTA (Sigma) in HBSS (without $\mathrm{Ca}^{2+}$ and $\mathrm{Mg}^{2+}$; Life Technologies) at $37^{\circ} \mathrm{C}$ in a 547 shaking water bath for $30 \mathrm{~min}$ (50 ml EDTA/HBSS per punch) (82). This incubation was 548 repeated three times with two washing steps (10 $\mathrm{min}$ in HBSS/antibiotics) after each 549 incubation period. Subsequently, mucosal specimens were cultured in a defined volume 550 of medium $(39,82)$ for $12 \mathrm{~h}$. After $12 \mathrm{~h}$ of organ culture, the supernatant was harvested, 551 centrifuged and used for incubation of PMN.

\section{Immunohistochemical staining}

554 Biopsies of colonic mucosa were taken from patients diagnosed with active state of 555 inflammatory bowel disease (IBD), i.e. ulcerative colitis (UC) or Crohn`s disease (CD). 556 Immunofluorescence staining was performed using $2 \mu \mathrm{m}$ sections of formalin-fixed, 557 paraffin embedded (FFPE) human colon tissue previously fixed in 4\% PBS-buffered 558 methanol-free formaldehyde (Brenzinger, Walldorf, Germany) for 2h. After 559 deparaffinization, rehydration and heat-mediated antigen retrieval blocking was 560 performed by incubation with an antibody diluent. Immunofluorescence staining was 561 performed using OPAL ${ }^{\mathrm{TM}}$ technology (PerkinElmer, Waltham, MA, USA). Procedures 562 were performed according to the manufacturer's instructions.

\section{Structured-illumination microscopy}

565 Cells were re-suspended in RPMI 1640 without FCS at a density of $1 \times 10^{6} \mathrm{cells} / \mathrm{ml}$.

566 These cells were seeded on poly-D-lysine (final conc.: 0.01mg/ml, Merck, Darmstadt, 567 Germany) coated coverslips $(\varnothing 10 \mathrm{~mm}, 1.5 \mathrm{H}$ high-precision, Marienfeld, Laud 568 Königshofen, Germany) and incubated for $15 \mathrm{~min}$ at $37^{\circ} \mathrm{C}$. Cells were fixed using $1.5 \%$ 569 PFA and stained as indicated in the figures. Coverslips were finally washed three times 570 with PBS containing $1 \% \mathrm{BSA}_{0} 0.1 \%$ saponin and $0.07 \% \mathrm{NaN}_{3}$ and finally with deionized 571 water. Coverslips were then mounted on a microscopy slide (ProLong Gold, Thermo 572 Fisher Scientific). 3D-SIM images were taken at latest two days after sample preparation 573 with a Nikon N-SIM microscope (Nikon, Tokio, Japan) using a 100x objective (SR Apo 574 TIRF100x NA 1.49). The reconstruction parameters were set as following: Illumination 575 Modulation Contrast: 0.08; High resolution noise suppression: 0.8; Out of focus blur 576 suppression: 0.8. If Illumination Modulation Contrast was set to auto-detection, the 
577 reconstruction quality was calculated between 7-8 (scale 1-10). Image analysis and

578 lookup table adjustments were performed using NIS-Elements V 4.30 (Nikon).

580 Confocal laserscan microscopy (NETosis detection)

581 Immunofluorescence staining were essentially done as described (31). Briefly, PMN 582 were plated on slides for 15 minutes. All following steps were performed at room 583 temperature. Cells were washed with PBS and fixed in 1.5\% paraformaldehyde in PBS 584 overnight. Cells were permeabilized with $0.3 \%$ Triton X-100 and stained with DAPI. 3D585 SIM images were taken within two days after sample preparation with a Nikon A1 586 microscope using a $20 x$ objective.

\section{Expression analysis}

589 Total RNA was isolated from rPMN and cPMN of three healthy blood donors. PMN were 590 isolated by gradient centrifugation followed by magnetic bead-based separation. Total 591 RNA was then purified from 10,000 cells using MagNA Pure (Promega, Mannheim, 592 Germany) in the inhouse molecular immunodiagnostic facility according to the 593 manufacturer's instructions. All RNA samples were quantified by using Qubit RNA assay 594 kit (Thermo Fisher Scientific, Waltham, Massachusetts), and RNA integrity was 595 assessed using Agilent 2100 Bioanalyzer system. Gene expression was analyzed in 596 collaboration with the NanoString Core facility of Heidelberg. Briefly, $25 \mu \mathrm{g}$ total RNA

597 (5 $4 \mathrm{~L} /$ sample) was mixed with $\mathrm{nCounter}{ }^{\circledR}$ reporter CodeSet $(3 \mu \mathrm{L})$ and $\mathrm{nCounter} \AA$ 598 capture ProbeSet $(2 \mu \mathrm{L})$ with hybridization buffer $(5 \mu \mathrm{L})$ for an overnight hybridization 599 reaction at $65^{\circ} \mathrm{C}$. The reaction was cooled down to $4{ }^{\circ} \mathrm{C}$, the samples were purified and 600 immobilised on a cartridge, and data was assessed on the nCounter SPRINT Profiler. 601 The exported data was analysed using Nanostring nSolver 4.0. For downstream 602 analysis, only genes with an expression value of $\geq 100$ CodeSet counts in all three 603 replicates of either rPMN or cPMN or both were considered. Differential expression 604 analysis was performed using limma with $P$ values adjusted for multiple comparisons 605 according to Benjamini and Hochberg, corresponding to a false discovery rate (83). 606 Genes with an expression of below this threshold were not further analysed (84). 607 Differentially expressed gene sets were mapped onto gene interaction networks from 608 STRING v.11 (85). STRING integrates protein-protein interactions from literature 
609 curation, computationally predicted interactions, and interactions transferred from model

610 organisms based on orthology.

611

612

613 Ethics statement

614 All human studies were approved by the ethics committee of the University of

615 Heidelberg (ethical votes S-024/2003, S-285/2015, S-390/2015 and S-119/2017) and

616 performed in accordance with the principles laid down in the Declaration of Helsinki.

617 Written informed consent was obtained from the patients.

618

619 Statistics

620 Statistical analysis was performed using GraphPad Prism V6 (La Jolla, CA, USA). For 621 experimental data, means and standard errors of the means (SEM) are shown. Two 622 groups are compared by two-sided t-tests and multiple comparisons by ANOVA and 623 Fisher's LSD test. Evaluation of PMN from the peripheral blood of IBD patients is 624 depicted as box plot and analyzed by Kruskal-Wallis test.

\section{Acknowledgements}

627 The authors thank Dr. Felix Lasitschka, Antje Heidtmann und Sabine Wendtrup for their 628 help in tissue preparation and staining, Prof. Dr. Maria Hänsch and PD Dr. Luise 629 Erpenbeck for critical reading of the manuscript draft, Ralph Röth and Heike Kuzan from 630 the nCounter Core Facility Heidelberg for performing nCounter analyses and related 631 services. There are no conflicts of interest. 


\section{Literature}

634 1. V. Brinkmann et al., Neutrophil extracellular traps kill bacteria. Science 303, 15326351535 (2004).

636 2. V. Delgado-Rizo et al., Neutrophil Extracellular Traps and Its Implications in Inflammation: An Overview. Front Immunol 8, 81 (2017).

638 3. F. V. S. Castanheira, P. Kubes, Neutrophils and NETs in modulating acute and chronic inflammation. Blood 133, 2178-2185 (2019).

6404 4. J. Cools-Lartigue et al., Neutrophil extracellular traps sequester circulating tumor cells and promote metastasis. J Clin Invest, (2013).

642 5. R. Khandpur et al., NETs are a source of citrullinated autoantigens and stimulate inflammatory responses in rheumatoid arthritis. Sci Transl Med 5, 178ra140 (2013).

644 6. T. N. Ellis, B. L. Beaman, Interferon-gamma activation of polymorphonuclear 645 neutrophil function. Immunology 112, 2-12 (2004).

646 7. F. Colotta, F. Re, N. Polentarutti, S. Sozzani, A. Mantovani, Modulation of 647 granulocyte survival and programmed cell death by cytokines and bacterial products. $648 \quad$ Blood 80, 2012-2020 (1992).

649 8. H. L. Wright, R. J. Moots, R. C. Bucknall, S. W. Edwards, Neutrophil function in 650 inflammation and inflammatory diseases. Rheumatology (Oxford) 49, 1618-1631 (2010). 9. N. Samadi et al., T-cell-derived cytokines enhance the antigen-presenting capacity of human neutrophils. Eur J Immunol 49, 1441-1443 (2019). 10. B. G. Yipp, P. Kubes, NETosis: how vital is it? Blood 122, 2784-2794 (2013). 11. R. Grieshaber-Bouyer, P. A. Nigrovic, Neutrophil Heterogeneity as Therapeutic Opportunity in Immune-Mediated Disease. Front Immunol 10, 346 (2019).

12. X. Xie et al., Single-cell transcriptome profiling reveals neutrophil heterogeneity in homeostasis and infection. Nat Immunol 21, 1119-1133 (2020). in Health and Disease. Trends Immunol 40, 565-583 (2019).

660 14. C. Iking-Konert et al., Polymorphonuclear neutrophils in Wegener's granulomatosis acquire characteristics of antigen presenting cells. Kidney Int 60, 22472262 (2001).

15. G. C. Spagnoli et al., Expression of HLA-DR in granulocytes of polytraumatized patients treated with recombinant human granulocyte macrophage-colony-stimulating factor. Hum Immunol 43, 45-50 (1995). acidosis promotes neutrophil transdifferentiation to $\mathrm{MHC}$ class II-expressing cells. Cell Immunol 271, 214-218 (2011). fluid neutrophils transcribe and express class II major histocompatibility complex molecules in rheumatoid arthritis. Arthritis Rheum 48, 2796-2806 (2003). dendritic-like cells at the site of inflammation in rheumatoid arthritis: evidence for activation by T cells. Ann Rheum Dis 64, 1436-1442 (2005).

19. M. S. Davey et al., Microbe-specific unconventional $\mathrm{T}$ cells induce human neutrophil differentiation into antigen cross-presenting cells. J Immunol 193, 3704-3716 (2014).

678 20. A. Takashima, Y. Yao, Neutrophil plasticity: acquisition of phenotype and 
21. C. Iking-Konert et al., Transdifferentiation of polymorphonuclear neutrophils: acquisition of CD83 and other functional characteristics of dendritic cells. $J$ Mol Med (Berl) 79, 464-474 (2001).

683 22. V. M. Kamp et al., Modulation of granulocyte kinetics by GM-CSF/IFN-gamma in a human LPS rechallenge model. $J$ Leukoc Biol 94, 513-520 (2013).

685 23. E. Jablonska, M. Kiluk, W. Markiewicz, J. Jablonski, Priming effects of GM-CSF, 686 IFN-gamma and TNF-alpha on human neutrophil inflammatory cytokine production. 687 Melanoma Res 12, 123-128 (2002).

688 24. N. T. Doncheva, J. H. Morris, J. Gorodkin, L. J. Jensen, Cytoscape StringApp: Network Analysis and Visualization of Proteomics Data. J Proteome Res 18, 623-632 690 (2019).

691 25. B. Chami, A. W. Yeung, C. van Vreden, N. J. King, S. Bao, The role of CXCR3 in DSS-induced colitis. PLoS One 9, e101622 (2014).

26. V. Langer et al., IFN-gamma drives inflammatory bowel disease pathogenesis through VE-cadherin-directed vascular barrier disruption. J Clin Invest 129, 4691-4707 (2019).

27. X. Han et al., Lactobacillus rhamnosus GG prevents epithelial barrier dysfunction induced by interferon-gamma and fecal supernatants from irritable bowel syndrome patients in human intestinal enteroids and colonoids. Gut Microbes 10, 59-76 (2019).

28. A. Y. Tilahun, M. Holz, T. T. Wu, C. S. David, G. Rajagopalan, Interferon gammadependent intestinal pathology contributes to the lethality in bacterial superantigeninduced toxic shock syndrome. PLoS One 6, e16764 (2011).

702

703

704

705

29. L. E. Hind, W. J. Vincent, A. Huttenlocher, Leading from the Back: The Role of the Uropod in Neutrophil Polarization and Migration. Dev Cell 38, 161-169 (2016).

30. E. Neubert et al., Chromatin swelling drives neutrophil extracellular trap release. Nat Commun 9, 3767 (2018).

31. E. Neubert et al., Serum and Serum Albumin Inhibit in vitro Formation of Neutrophil Extracellular Traps (NETs). Front Immunol 10, 12 (2019).

32. N. Krishnamoorthy et al., Neutrophil cytoplasts induce TH17 differentiation and skew inflammation toward neutrophilia in severe asthma. Sci Immunol 3, (2018).

33. M. G. Duvall, N. Krishnamoorthy, B. D. Levy, Non-type 2 inflammation in severe asthma is propelled by neutrophil cytoplasts and maintained by defective resolution. Allergol Int 68, 143-149 (2019).

34. W. Zhao, D. K. Fogg, M. J. Kaplan, A novel image-based quantitative method for the characterization of NETosis. J Immunol Methods 423, 104-110 (2015).

35. Z. Fonseca et al., Entamoeba histolytica Induce Signaling via Raf/MEK/ERK for Neutrophil Extracellular Trap (NET) Formation. Front Cell Infect Microbiol 8, 226 (2018). 36. D. X. Nguyen, M. R. Ehrenstein, Anti-TNF drives regulatory T cell expansion by paradoxically promoting membrane TNF-TNF-RIl binding in rheumatoid arthritis. J Exp Med 213, 1241-1253 (2016).

37. M. Wong et al., TNFalpha blockade in human diseases: mechanisms and future directions. Clin Immunol 126, 121-136 (2008).

38. A. D. Levin, M. E. Wildenberg, G. R. van den Brink, Mechanism of Action of AntiTNF Therapy in Inflammatory Bowel Disease. J Crohns Colitis 10, 989-997 (2016).

39. J. Schroder-Braunstein et al., Initiation of an inflammatory response in resident intestinal lamina propria cells -use of a human organ culture model. PLoS One 9, e97780 (2014). 
40. E. Hawila et al., CCR5 Directs the Mobilization of CD11b(+)Gr1(+)Ly6C(low) Polymorphonuclear Myeloid Cells from the Bone Marrow to the Blood to Support Tumor Development. Cell Rep 21, 2212-2222 (2017).

41. A. Ariel et al., Apoptotic neutrophils and T cells sequester chemokines during immune response resolution through modulation of CCR5 expression. Nat Immunol 7, 1209-1216 (2006). neutrophil apoptosis and regulation of cell survival by granulocyte macrophage-colony stimulating factor. J Leukoc Biol 78, 1408-1418 (2005).

43. P. Li, A. T. Bademosi, J. Luo, F. A. Meunier, Actin Remodeling in Regulated Exocytosis: Toward a Mesoscopic View. Trends Cell Biol 28, 685-697 (2018).

44. K. R. McLeish et al., Exocytosis of neutrophil granule subsets and activation of prolyl isomerase 1 are required for respiratory burst priming. $J$ Innate Immun 5, 277-289 (2013).

45. T. Mitchell, A. Lo, M. R. Logan, P. Lacy, G. Eitzen, Primary granule exocytosis in human neutrophils is regulated by Rac-dependent actin remodeling. Am J Physiol Cell Physiol 295, C1354-1365 (2008).

46. H. R. Thiam et al., NETosis proceeds by cytoskeleton and endomembrane disassembly and PAD4-mediated chromatin decondensation and nuclear envelope rupture. Proc Natl Acad Sci U S A 117, 7326-7337 (2020).

47. K. D. Metzler, C. Goosmann, A. Lubojemska, A. Zychlinsky, V. Papayannopoulos, A myeloperoxidase-containing complex regulates neutrophil elastase release and actin dynamics during NETosis. Cell Rep 8, 883-896 (2014).

750 is a cell surface cytotoxic transmembrane protein: ramifications for the complex physiology of TNF. Cell 53, 45-53 (1988).

49. Z. Szondy, A. Pallai, Transmembrane TNF-alpha reverse signaling leading to TGF-beta production is selectively activated by TNF targeting molecules: Therapeutic implications. Pharmacol Res 115, 124-132 (2017).

50. S. Ardestani et al., Membrane versus soluble isoforms of TNF-alpha exert opposing effects on tumor growth and survival of tumor-associated myeloid cells. Cancer Res 73, 3938-3950 (2013).

759

760 51. W. J. Sandborn et al., Etanercept for active Crohn's disease: a randomized, double-blind, placebo-controlled trial. Gastroenterology 121, 1088-1094 (2001).

52. J. Korzenik, M. D. Larsen, J. Nielsen, J. Kjeldsen, B. M. Norgard, Increased risk of developing Crohn's disease or ulcerative colitis in 17018 patients while under treatment with anti-TNFalpha agents, particularly etanercept, for autoimmune diseases other than inflammatory bowel disease. Aliment Pharmacol Ther 50, 289-294 (2019).

53. A. Nesbitt et al., Mechanism of action of certolizumab pegol (CDP870): in vitro comparison with other anti-tumor necrosis factor alpha agents. Inflamm Bowel Dis 13, 1323-1332 (2007).

54. R. Atreya et al., Antibodies against tumor necrosis factor (TNF) induce T-cell apoptosis in patients with inflammatory bowel diseases via TNF receptor 2 and intestinal CD14(+) macrophages. Gastroenterology 141, 2026-2038 (2011).

55. T. ten Hove, C. van Montfrans, M. P. Peppelenbosch, S. J. van Deventer, Infliximab treatment induces apoptosis of lamina propria $\mathrm{T}$ lymphocytes in Crohn's disease. Gut 50, 206-211 (2002). 
56. A. C. Vos et al., Regulatory macrophages induced by infliximab are involved in healing in vivo and in vitro. Inflamm Bowel Dis 18, 401-408 (2012).

57. L. Vangelista, S. Vento, The Expanding Therapeutic Perspective of CCR5 Blockade. Front Immunol 8, 1981 (2017).

58. I. Cleynen et al., Inherited determinants of Crohn's disease and ulcerative colitis phenotypes: a genetic association study. Lancet 387, 156-167 (2016).

59. L. Jostins et al., Host-microbe interactions have shaped the genetic architecture of inflammatory bowel disease. Nature 491, 119-124 (2012).

60. M. B. Dwinell, L. Eckmann, J. D. Leopard, N. M. Varki, M. F. Kagnoff, Chemokine receptor expression by human intestinal epithelial cells. Gastroenterology 117, 359-367 (1999).

61. A. Mencarelli et al., Highly specific blockade of CCR5 inhibits leukocyte trafficking and reduces mucosal inflammation in murine colitis. Sci Rep 6, 30802 (2016).

62. M. N. Ajuebor, C. M. Hogaboam, S. L. Kunkel, A. E. Proudfoot, J. L. Wallace, The chemokine RANTES is a crucial mediator of the progression from acute to chronic colitis in the rat. $J$ Immunol 166, 552-558 (2001).

63. A. J. Flegler, G. C. Cianci, T. J. Hope, CCR5 conformations are dynamic and modulated by localization, trafficking and G protein association. PLoS One 9, e89056 (2014).

64. J. Lemay, S. Marullo, R. Jockers, M. Alizon, A. Brelot, On the dimerization of CCR5. Nat Immunol 6, 535; author reply 535-536 (2005).

65. C. Blanpain et al., Multiple active states and oligomerization of CCR5 revealed by functional properties of monoclonal antibodies. Mol Biol Cell 13, 723-737 (2002).

66. O. Wera, P. Lancellotti, C. Oury, The Dual Role of Neutrophils in Inflammatory Bowel Diseases. J Clin Med 5, (2016).

67. P. J. Trivedi, D. H. Adams, Chemokines and Chemokine Receptors as Therapeutic Targets in Inflammatory Bowel Disease; Pitfalls and Promise. J Crohns Colitis 12, 1508 (2018).

68. V. Dinallo et al., Neutrophil Extracellular Traps Sustain Inflammatory Signals in Ulcerative Colitis. J Crohns Colitis 13, 772-784 (2019).

69. Z. Kubiritova, J. Radvanszky, R. Gardlik, Cell-Free Nucleic Acids and their Emerging Role in the Pathogenesis and Clinical Management of Inflammatory Bowel Disease. Int J Mol Sci 20, (2019).

70. I. Angelidou et al., REDD1/Autophagy Pathway Is Associated with NeutrophilDriven IL-1beta Inflammatory Response in Active Ulcerative Colitis. J Immunol 200, 3950-3961 (2018).

71. D. Muthas et al., Neutrophils in ulcerative colitis: a review of selected biomarkers and their potential therapeutic implications. Scand J Gastroenterol 52, 125-135 (2017).

72. J. Albrengues et al., Neutrophil extracellular traps produced during inflammation awaken dormant cancer cells in mice. Science 361, (2018).

73. R. Barry et al., Faecal neutrophil elastase-antiprotease balance reflects colitis severity. Mucosal Immunol 13, 322-333 (2020).

74. E. Kiner et al., Gut CD4(+) T cell phenotypes are a continuum molded by microbes, not by TH archetypes. Nat Immunol 22, 216-228 (2021).

75. L. G. Ng, R. Ostuni, A. Hidalgo, Heterogeneity of neutrophils. Nat Rev Immunol 19, 255-265 (2019). 
820 76. R. Grieshaber-Bouyer et al., The neutrotime transcriptional signature defines a single continuum of neutrophils across biological compartments. Nat Commun 12, 2856 822 (2021).

823 77. J. Pillay et al., A subset of neutrophils in human systemic inflammation inhibits T cell responses through Mac-1. J Clin Invest 122, 327-336 (2012).

78. G. Wabnitz, H. Kirchgessner, Y. Samstag, Qualitative and Quantitative Analysis of the Immune Synapse in the Human System Using Imaging Flow Cytometry. J Vis 827 Exp, (2019).

828 79. G. H. Wabnitz, H. Kirchgessner, Y. Samstag, Imaging Flow Cytometry for

829 Multiparametric Analysis of Molecular Mechanism Involved in the Cytotoxicity of Human 830 CD8+ T-cells. J Cell Biochem 118, 2528-2533 (2017).

831 80. E. Balta et al., Spatial oxidation of L-plastin downmodulates actin-based functions 832 of tumor cells. Nat Commun 10, 4073 (2019).

833 81. V. Dominical, L. Samsel, J. P. McCoy, Jr., Masks in imaging flow cytometry. 834 Methods 112, 9-17 (2017).

835 82. T. Szikszai et al., Standardization of a human organ culture model of intestinal 836 inflammation and its application for drug testing. $J$ Immunol Methods 421, 96-103 837 (2015).

838 83. Y. Benjamini, Y. Hochberg, Controlling the False Discovery Rate: A Practical and 839 Powerful Approach to Multiple Testing. Journal of the Royal Statistical Society. Series B 840 (Methodological) 57, 289-300 (1995).

841 84. $\mathrm{H}$. Wang et al., NanoStringDiff: a novel statistical method for differential 842 expression analysis based on NanoString nCounter data. Nucleic Acids Res 44, e151 843 (2016).

844 85. D. Szklarczyk et al., STRING v11: protein-protein association networks with 845 increased coverage, supporting functional discovery in genome-wide experimental 846 datasets. Nucleic Acids Res 47, D607-D613 (2019). 


\section{Figure 1}

852 Cytokine stimulation of human PMN induces the development of a CCR5853 expressing PMN subgroup (CCR5 ${ }^{+}$CPMN)

854 A) Whole blood PMN (WB-PMN) from healthy individuals were purified by density 855 gradient centrifugation followed by negative magnetic bead isolation obtaining resting 856 PMN (rPMN). These cells were activated by the cytokines GM-CSF (100 U/ml) and IFN $\gamma$ $857(10 \mathrm{ng} / \mathrm{ml})$ for two days resulting in cytokine-incubated PMN (cPMN) that were used for 858 RNA isolation.

859 B) Heatmap of NanoString nCounter-based (NanoString Technologies, Inc.) comparing 860 rPMN and cPMN of three different blood donors. Only genes with an expression value of $861 \geq 100$ CodeSet counts in all three replicates in one group were considered.

862 C) Interaction network among up (left) and down (right) regulated genes found in the 863 nCounter analysis of rPMN and cPMN. The graph was generated from STRING 864 database.

865 Connections reflect physical protein interactions. The determination of the connection is 866 shown below as color code. A small protein-protein interaction (PPI) enrichment $p$-value 867 indicate that the nodes are not random and that the observed number of edges is 868 significant.

869 D) The surface expression of chemokine receptors in rPMN (open histograms) and 870 cPMN (tinted histograms) was analyzed using flow cytometry. The autofluorescence of 871 cPMN is shown as dotted histogram. The histograms are representative for three 872 independent experiments.

873 E-F) CCR 1 and CCR5 co-expression was analyzed on CPMN by (double-staining) flow 874 cytometry (E, dot plot). The amount of $\mathrm{CCR}^{+} \mathrm{CPMN}$ of total PMN was quantified and is 875 depicted for 20 independent experiments in $\mathbf{F}\left(\mathrm{n}=20\right.$; ANOVA; $\left.{ }^{* *} \mathrm{p}<0.01\right)$.

876 G) The percentage of $\mathrm{CCR}^{+} \mathrm{cPMN}$ was assessed after two days of incubation of 877 primary human PMN with the indicated concentrations of GM-CSF and IFN $\gamma(n=4)$.

$878 \mathrm{H}$ ) Percentage of $\mathrm{CCR} 5^{+} \mathrm{cPMN}$ was quantified at day 0 ( $3 \mathrm{~h}$ after adding cytokines) and 879 at days 1-3 using flow cytometry. Statistics represent ANOVA test with d0 as control 880 value 
(SEM; $\left.n=4 ;{ }^{* *} p<0.05 ;{ }^{* *} p<0.01\right)$.

\section{Figure 2}

884 CCR5 is newly expressed and expelled from intracellular stores

885 A-B) Total CCR5 expression was detected in whole cell lysates from rPMN and cPMN 886 by western blot analysis. GAPDH immunostaining served as loading control. The blot in $887 \mathrm{~A}$ is representative for three independent experiments. A quantification of the CCR5 888 expression as ratio between CCR5- and GAPDH-derived signals is depicted in B (SEM; 889 n=3; t-test; n.s.=not significant).

890 C) CCR5 was detected on rPMN (left) and cPMN (right) by surface immunostaining 891 (upper part) or by total immunostaining (lower part) using $0.1 \%$ saponin to permeabilize 892 the cells. Shown are representative flow cytometry histograms taken from three 893 independent experiments.

894 D) rPMN and cPMN were adhered on coverslips, fixed, permeabilized and 895 immunostained for CD66b (blue) and CCR5 (red). In addition F-actin (phalloidin-AF488) 896 and nuclei (DAPI) were labelled. Images of 28 cells from three independent experiments 897 were acquired using structured-illumination microscopy (3D-SIM).

898 E) rPMN and cPMN were fixed and stained with phalloidin-AF488 to quantify F-actin by 899 imaging flow cytometry. Shown is the total intensity from 6 independent experiments $900 \quad\left(\mathrm{n}=6\right.$, SEM, ANOVA, $\left.{ }^{*} \mathrm{p}<0.05,{ }^{* *} \mathrm{p}<0.01\right)$.

901 F) PMN were stimulated with GM-CSF/ IFN $\gamma$ in the presence or absence of either CytoD 902 (250nM) or Jas (500nM) for one day. The expression of CCR5 was quantified using flow 903 cytometry $\left(n=3\right.$, SEM, ANOVA, $\left.{ }^{*} p<0.05,{ }^{* *} p<0.01\right)$.

904 G-H) The expression of PMN lineage-associated receptors (G), CD66b, CD15, CD62L, 905 CD16, HLA-DR and CD49d) as well as chemokine receptors (H), CXCR1, CXCR3, 906 CXCR4, CCR3, CCR4) were analyzed on rPMN, CCR5 and CCR5 ${ }^{+} \mathrm{cPMN}^{-}$by flow 907 cytometry. The cells were gated for $\mathrm{CD} 6 \mathrm{~b}^{+}$(rPMN) and their expression of CCR5 908 (CD66b $\left.{ }^{+} \mathrm{cPMN}\right)$. The histograms are representative for three independent experiments. 
913 A) cPMN were stimulated with SDF1 $\alpha(100 \mathrm{ng} / \mathrm{ml})$ or CCL5 $(100 \mathrm{ng} / \mathrm{ml})$ for 5 minutes.

914 Then, cells were fixed and stained for F-actin (green) and CCR5 (red). Images of 25,000

915 cells were acquired using imaging flow cytometry. Shown are representative images of

$916 \mathrm{CCR5}^{-}$cPMN (left) and CCR5 ${ }^{+}$cPMN (right) that were either left untreated (upper panel)

917 or stimulated as indicated (lower panels). The arrows indicate polarized F-actin as it

918 occurs after cell polarization.

919 B) Quantification of the percentage of polarized CCR5 (white bars) and CCR5 ${ }^{+} \mathrm{CPMN}^{-}$

920 (black bars) after treatment with solvent control (Ctr.), SDF1 $\alpha$ or CCL5 is shown ( $n=3$,

921 SEM, ANOVA, $\left.{ }^{*} \mathrm{p}<0.05,{ }^{* *} \mathrm{p}<0.01\right)$.

922 C) Migration of PMN was performed in a transwell assay. Migrated cells were counted 923 using flow cytometry and immunostaining for CCR5. Migrated cells are depicted as 924 percent of the number of input cells of the respective subgroup $(n=3, S E M$, ANOVA, $92{ }^{* * *} \mathrm{p}<0.01$, n.s. $=$ not significant).

926 D) rPMN or cPMN were stimulated for one hour with fMLP or ionomycin. Afterwards, the 927 expression level of CD66b was measured for rPMN (left graph), CCR5 cPMN (middle 928 graph) and $\mathrm{CCR}^{+}$cPMN (right graph) by flow cytometry and normalized to time point 929 zero of the respective kinetics. Shown are the percentage changes as mean value from

9305 experiments $\left(n=5\right.$; SEM; ANOVA with $t=0$ min as control; ${ }^{*} p<0.05,{ }^{* *} p<0.01$ and $931{ }^{* * *} \mathrm{p}<0.001$ of ionomycin; \#\# $\mathrm{p}<0.01$ of $\left.\mathrm{fMLP}\right)$.

932 E-F) Phagocytosis of $E$. coli was analyzed for rPMN, CCR5 ${ }^{-} \mathrm{cPMN}$ and $\mathrm{CCR}^{+} \mathrm{cPMN}$ 933 using flow cytometry. Sample dot plots are shown in $\mathbf{E}$ and a quantification of PMN that 934 phagocytosed $E$. coli is shown in $\mathbf{F}\left(n=5\right.$; ANOVA, SEM, $\left.{ }^{*} p<0.05 ;{ }^{* *} p<0.01\right)$.

\section{Figure 4}

937 CCL5 induced ROS production accelerating NETosis of pro-NETotic CCR5 ${ }^{+}$CPMN

938 A) Flow cytometric analysis of cPMN before magnet-bead sorting, and the negatively 939 sorted CCR5 $^{-}$cPMN and positively sorted $\mathrm{CCR}^{+}$cPMN. The respective

940 autofluorescence is shown as an open histogram. The figure is representative for 3 941 independent experiments.

942 B) Microscopic confocal laser-scanned images of sorted CCR5 cPMN (upper panel) 943 and purified CCR5+ cPMN (lower panels). While CCR5 cPMN mostly show a lobed 944 nucleus, different nuclear morphologies can be seen in CCR5 ${ }^{+} \mathrm{cPMN}$. The percentage 
945 of each morphology is shown on the right side and represents 3 independent 946 experiments.

947 C) Nuclear morphology was analyzed by imaging flow cytometry. PMN were fixed and 948 stained with fluorescently labelled antibodies against CD66b (blue) and CCR5 (green) 949 as well as with DAPI (red) to visualize nuclei. The images are representative for 6 950 independent experiments in which 25,000 cells were acquired.

951 D) Nuclear circularity was quantified for rPMN (dotted line) and CCR5 ${ }^{-}$cPMN (solid line) 952 and $\mathrm{CCR}^{+}$cPMN (grey histogram) by imaging flow cytometry. Data are representative 953 for 6 independent experiments. A quantification is shown in the graph on the right $(n=6$;

954 SEM, ANOVA, $\left.{ }^{* *} \mathrm{p}<0.01\right)$

955 E) To quantify NETosis, the chromatin-pattern was analyzed by imaging flow cytometry. 956 A NETotic phenotype is present if the DNA-sharpness ( $y$-axis) is small and at the same 957 time the area on which the chromatin is distributed increases (x-axis). Shown are 958 percent NETotic PMN in rPMN (left dot plot), CCR5 ${ }^{-}$cPMN (middle dot plot) and CCR5 ${ }^{+}$ 959 cPMN (right dot plot). The graph on the right show the mean percentages of NETotic 960 PMN either unstimulated or after PMA-treated for $45 \mathrm{~min}(\mathrm{n}=3$; SEM; ANOVA, * $\mathrm{p}<0.05)$.

961 F) Cytoplasts can be identified due to their depleted DNA content as measured by the 962 intensity of DAPI-derived fluorescence of the cells. The sample dot plots and the 963 quantification is analogous to the presentation in $C\left(n=3\right.$; SEM; ANOVA, $\left.{ }^{*} p<0.05\right)$.

964 G) cPMN incubated with CCL5 alone or together with the indicated reagents and 965 incubated for $2 \mathrm{~h}$. The nuclear morphology was determined by imaging flow cytometry as 966 described above ( $n=3$; SEM; ANOVA, $\left.{ }^{*} \mathrm{p}<0.05 ;{ }^{* *} \mathrm{p}<0.01\right)$.

967 H) To determine the intracellular ROS level, cPMN were loaded with CellROX ${ }^{\circledR}$ green 968 and stimulated with CCL5 for $5 \mathrm{~min}$. The flow cytometric histogram shows CellROX ${ }^{\circledR}$ 969 green in unstimulated cells ( $\mathrm{t}=0 \mathrm{~min}$, open histogram) and CCL5-treated cells ( $\mathrm{t}=5 \mathrm{~min}$, 970 closed histogram). The numbers indicate the geo-mean values, obtained from the 971 histograms in log-scale. The figure is representative for three independent experiments.

972 I) The changes in ROS level after CCL5 treatment are shown as kinetics. Values are 973 presented relative to unstimulated cells, which were set to $100 \%(n=3$; SEM; ANOVA, $\left.974{ }^{*} \mathrm{p}<0.05 ;{ }^{* * *} \mathrm{p}<0.001\right)$ 


\section{Increased ELANE expression in CCR5 ${ }^{+}$cPMN}

978 A-B) The expression of ELANE was quantified by intracellular fluorescent

979 immunolabelling. The grey histogram (A) shows the elevated ELANE level in CCR5 ${ }^{+}$

980 PMN, while the ELANE expression of CCR5 ${ }^{-}$PMN (open histogram) and rPMN (dotted

981 histogram) was on a comparable level. A statistical evaluation of three independent

982 experiments is shown in $\mathbf{B}\left(\mathrm{n}=3\right.$; SEM; ANOVA, $\left.{ }^{*} \mathrm{p}<0.05\right)$.

983 C-E) The subcellular localization of ELANE was analyzed using imaging flow cytometry.

984 A co-localization of ELANE (green) and DAPI (red) appears in yellow in $\mathrm{CCR}^{+} \mathrm{cPMN}$

985 (C). Nuclear localization of ELANE was quantified by calculation of the similarity score 986 provided by imaging flow cytometry (D) and statistics were calculated for three 987 independent experiments (E) ( $n=3$; SEM; t-test, $\left.{ }^{*} p<0.05\right)$.

989 Figure 6

990 Membrane-bound TNF $\alpha$ mediates ELANE expression via TNFR2 and PI3K

991 A) Intracellular (TNF $\alpha$ ) and surface (TNFR1, TNFR2) expression of TNF $\alpha$ and its 992 receptors was analyzed on rPMN and cPMN by flow cytometry. The respective 993 autofluorescence was subtracted from the respective MFI values to obtain the $\Delta \mathrm{MFI}$.

994 The quantification shown below includes three independent experiments $(n=3, S E M$, 995 ANOVA, $\left.{ }^{*} \mathrm{p}<0.05 ;{ }^{* * *} \mathrm{p}<0.001\right)$.

996 B) ELANE upregulation was measured in rPMN and CCR5 ${ }^{+} \mathrm{cPMN}$. During the cytokine997 dependent diversification process, cells were incubated with or without the PI3K inhibitor 998 Ly294003 (Ly), an anti-TNF antibody (aTNF) or the NF-kB inhibitor TPCA ( $\mathrm{n}=3$; SEM; 999 ANOVA, $\left.{ }^{* *} \mathrm{p}<0.01\right)$

1000 C) PMN were incubated with either Ly294002, aTNF or TPCA during the diversification 1001 process. Subsequently, NETosis was detected by imaging flow cytometry $(n=3, S E M$, 1002 ANOVA, $\left.{ }^{*} \mathrm{p}<0.05\right)$.

1003 D) PMN were incubated with GM-CSF plus IFN $\gamma$ or TNF for two days and ELANE 1004 expression was determined by flow cytometry cytometry ( $n=4$, SEM, ANOVA, * $p<0.05)$.

1005 E) To investigate the localization of TNF in cPMN, cells were fixed, permeabilized, and 1006 treated with fluorescently labeled antibodies against TNF. Subsequently, the cells were 1007 analyzed by imaging flow cytometry. Typical images of rPMN, CCR5 ${ }^{-}$and $\mathrm{CCR}^{+} \mathrm{cPMN}^{\mathrm{c}}$ 1008 are shown in the upper panel. In the lower part, the left histogram shows the 
1009 internalization score of TNF and the right part shows the MFI of TNF on the membrane.

1010 Due to low expression, rPMN have not been evaluated here. The figure is representative 1011 of 3 experiments with different blood donors.

1012 F) Membrane-bound TNF was detected using fluorescently labelled antibodies that were 1013 applied to unpermeabilized cPMN (surface stain). The bar graph shows the statistical 1014 evaluation of three independent experiments $\left(n=3\right.$, SEM, t-test, $\left.{ }^{*} p<0.05\right)$.

1015 G) After incubation with GM-CSF plus IFN $\gamma$ for two days, the resulting cPMN were 1016 labeled with CellROX and then treated with CCL5 or antibodies directed against TNF 1017 (aTNF) for 5 minutes. ROS content was determined by flow cytometry ( $n=3$, SEM, 1018 ANOVA, $\left.{ }^{*} \mathrm{p}<0.05\right)$.

1019 H) After cytokine-induced diversification, cells were incubated with CCL5 or aTNFa for 2 1020 hours. Subsequently, NETosis was measured by imaging flow cytometry ( $n=3$, SEM, 1021 ANOVA, $\left.{ }^{*} \mathrm{p}<0.05\right)$.

1024 Frequency of $\mathbf{C C R}^{+}$CPMN ulcerative colitis patients

1025 A) Tissue sections of mucosal specimens (CG, UC and CD) were subject to 1026 immunofluorescence staining of azurocidine (green) and CCR5 (red). Nuclei were 1027 counterstained with DAPI (blue). The arrows indicate CCR5 ${ }^{+}$cPMN in colonic mucosa of 1028 UC patients. Shown are the results from one representative experiment out of three 1029 independent experiments per condition.

1030 B) The bar graph shows the quantification of total PMN (grey) and CCR5 ${ }^{+} \mathrm{cPMN}$ (black) 1031 per optical field. In total 15 optical fields were quantified from three different patients 1032 ( $\mathrm{n}=3$; SEM; ANOVA, ${ }^{*} \mathrm{p}<0.05,{ }^{*} \mathrm{p}<0.01$ of total PMN; \#\#\# $\mathrm{p}<0.01$ of CCR5 $\left.{ }^{+} \mathrm{cPMN}\right)$.

1033 C) Detection of the frequency of $\mathrm{CCR}^{+} \mathrm{cPMN}$ in rPMN or cells that were either 1034 stimulated with GM-CSF plus IFN $\gamma$ (GI) or with LEL supernatants ( $n=3$, SEM, ANOVA, $\left.1035{ }^{*} p<0.05 ;{ }^{* *} p<0.01\right)$.

1036 D) The frequency of $\mathrm{CCR}^{+} \mathrm{CPMN}$ was quantified in the peripheral blood of a control 1037 group (CG) or UC and CD patients (SEM; Kruskal-Wallis, ${ }^{*} p<0.01,{ }^{* * *} p<0.001$ ).

1038 E) A linear correlation of serum CRP level, the disease score (HBI: Harvey-Bradshaw 1039 Index or SCCAI: simple clinical colitis activity index) or fecal calprotectin level and the 
1040 frequency of $\mathrm{CCR}^{+} \mathrm{cPMN}$ in the whole blood was calculated for the patients shown in 1041 E.

1042 F) The graph shows the percentage of $\mathrm{CCR}^{+} \mathrm{cPMN}$ in the whole blood of UC and CD

1043 patients that were naïve for anti-TNF $\alpha$ therapy (white column, black dots), were 1044 successfully treated with infliximab or adalimumab (white column, green dots) and 1045 patients in which the infliximab or adalimumab therapy failed (grey column, black dots).

\section{$1047 \quad$ Figure 8}

1048 Three hit model of NETosis in inflammation

1049 A Surface CCR5 expression is initiated on PMN by exocytosis after dissassambly of the

1050 F-actin barrie as part of a either priming, stress response or apoptosis. Without survival/ 1051 anti-apoptotic signals PMN undergo apoptosis at this stage.

1052 B) Enhanced CCR5 expression by de novo synthesis is induced and anti-apoptotic 1053 mechansism are initiated (not shown). In addition, a process comprising three hits drives

1054 PMN into NETosis. In the first hit, PMN are stimulated by IFN $\gamma$ leading to an expression 1055 of TNF (left part). In the second hit, ELANE is overexpressed via a TNFR2 signaling 1056 pathway (middle part). TNF may have several sources. One is the $\mathrm{CCR}^{+} \mathrm{cPMN}$ by 1057 auto/paracrine stimulation via sTNF or mTNF. The third hit leads to activation of ELANE 1058 and induction of NETosis by ROS (right part). The latter can occur through stimulation of 1059 CCR5 with natural ligands.

1060 C) Blocking TNF antibodies can slow NETosis process by interfering with 1061 overexpression of ELANE downstrem of TNFR2.

1062 D) Antibodies to TNF can also induce an outside-in signal via mTNF. This produces 1063 ROS, which is an important mediator of NETosis. One function of ROS is to release 1064 ELANE from azurosomes, which accelerated NETosis. 
Figure 1

A

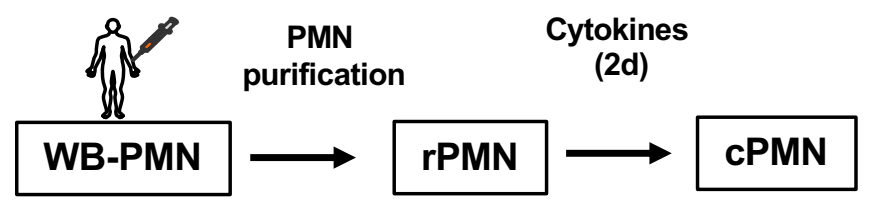

B

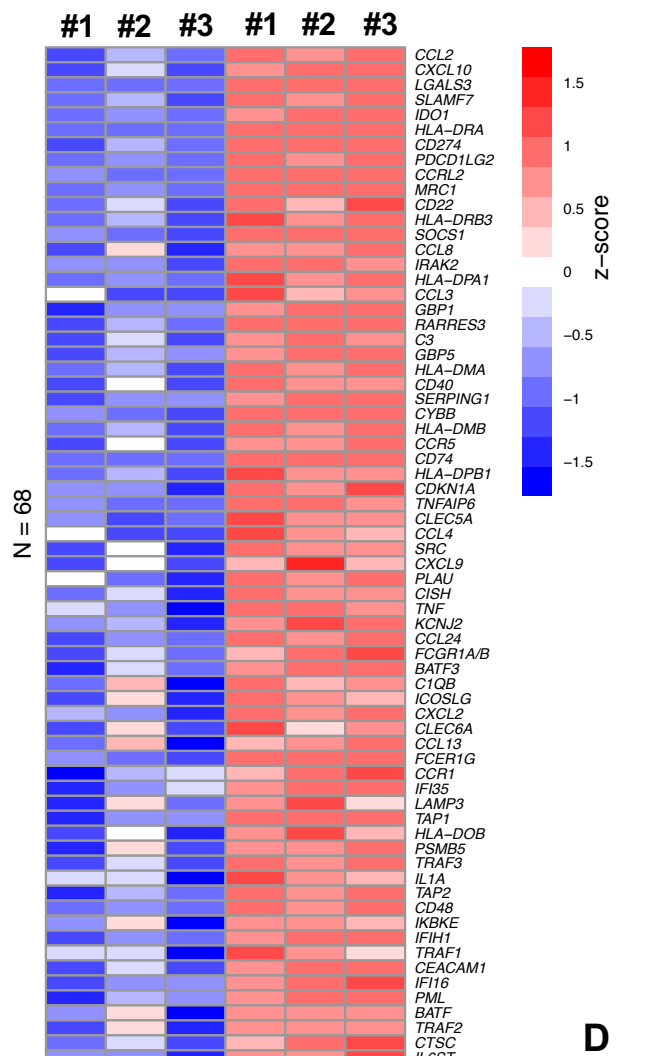

C Up-regulated genes

Down-regulated genes
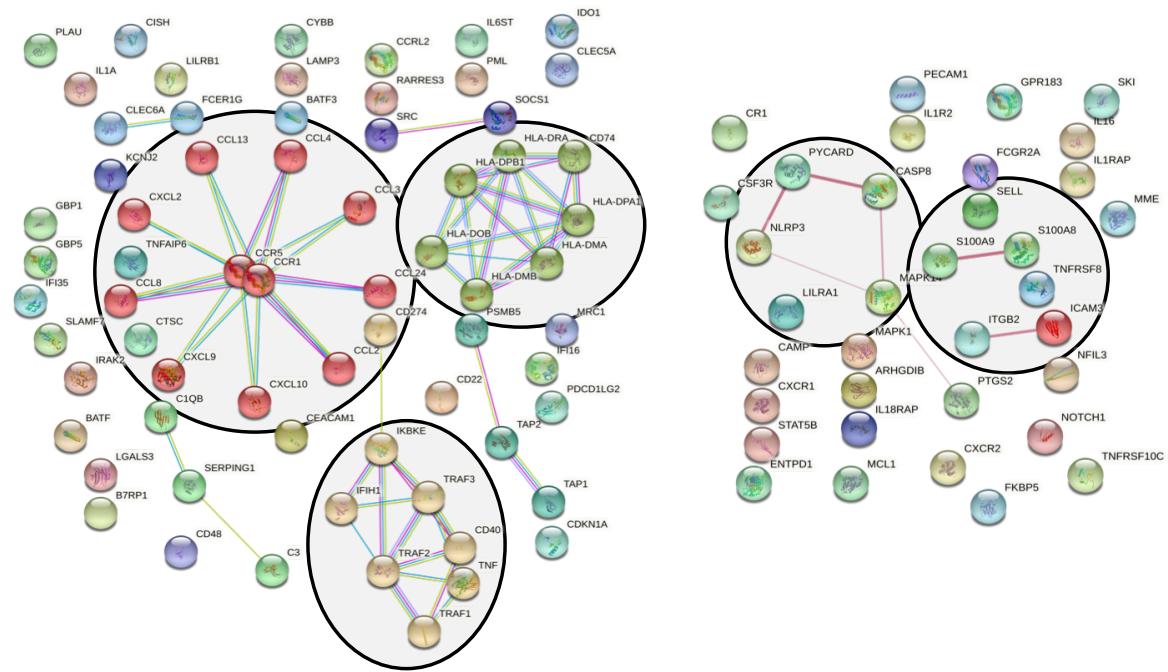

PPI enrichment $p$-value: $<1.0 \mathrm{e}-16$

PPI enrichment p-value: 0.0004

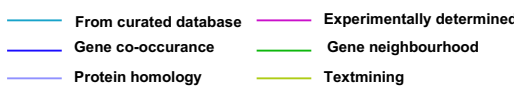

- Gene fusion

D
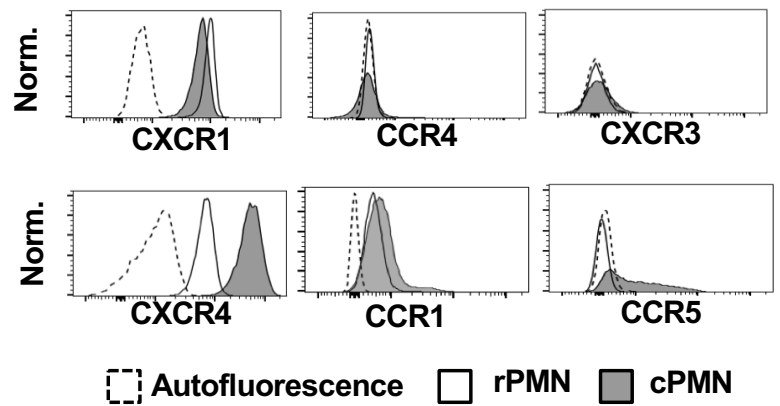

E

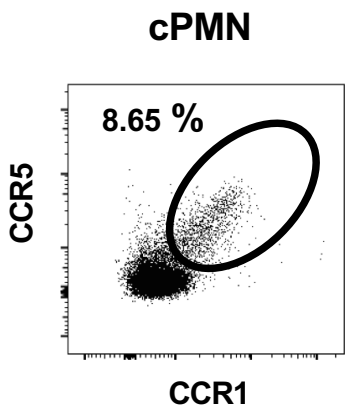

$\mathbf{F}$

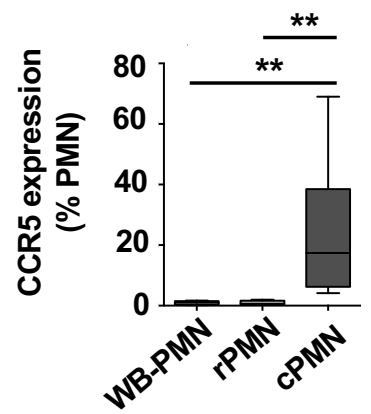

G

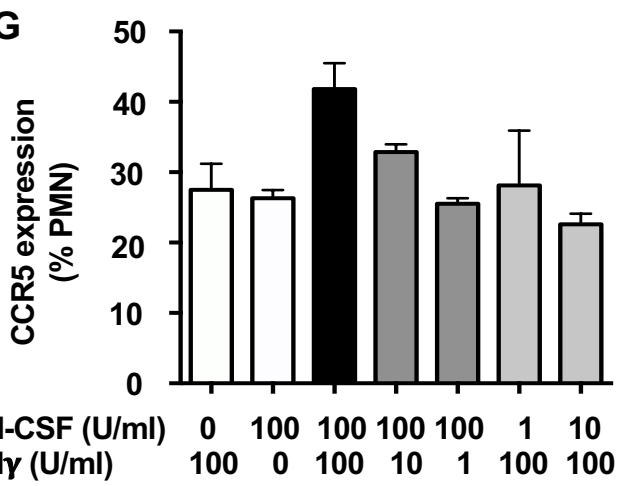

H

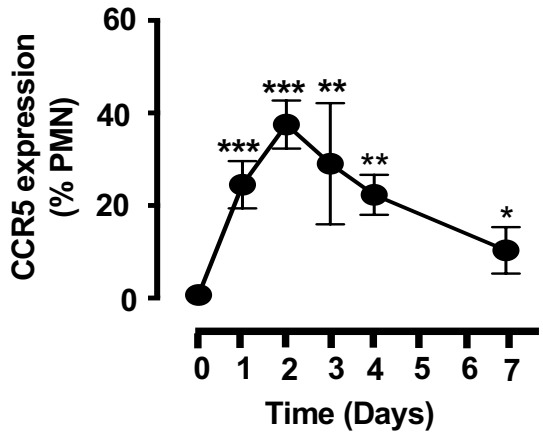


Figure 2

A

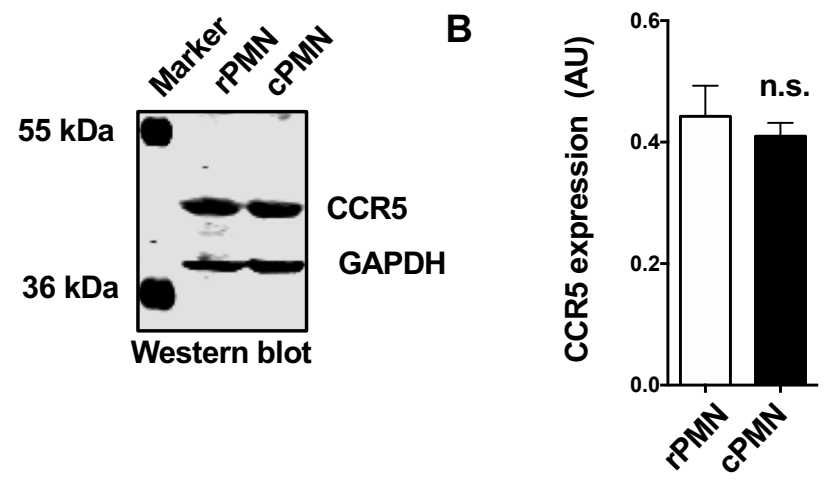

C

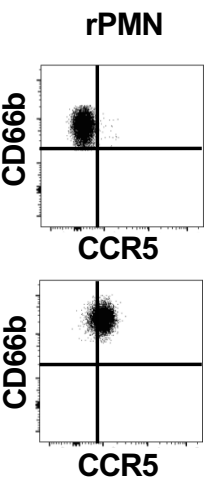

CPMN

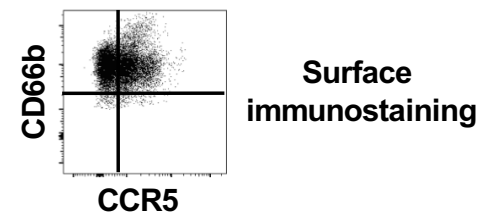

Total

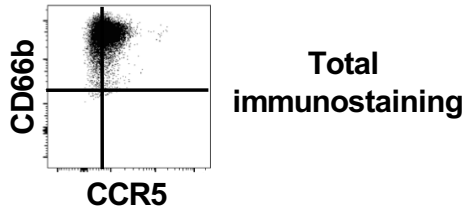

D

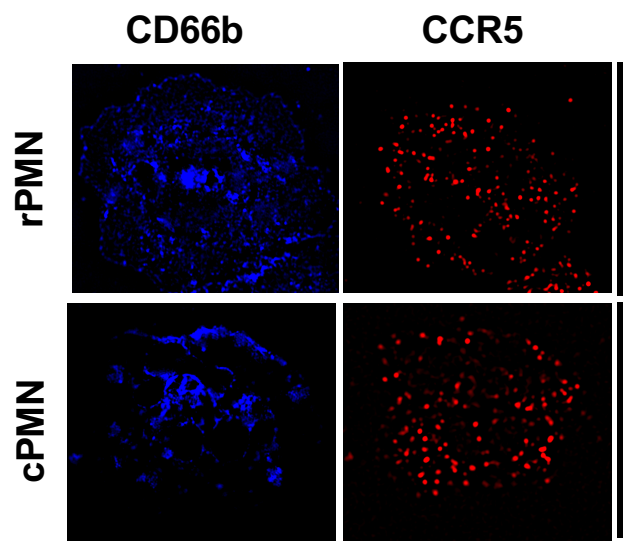

Structured Illumination microscopy
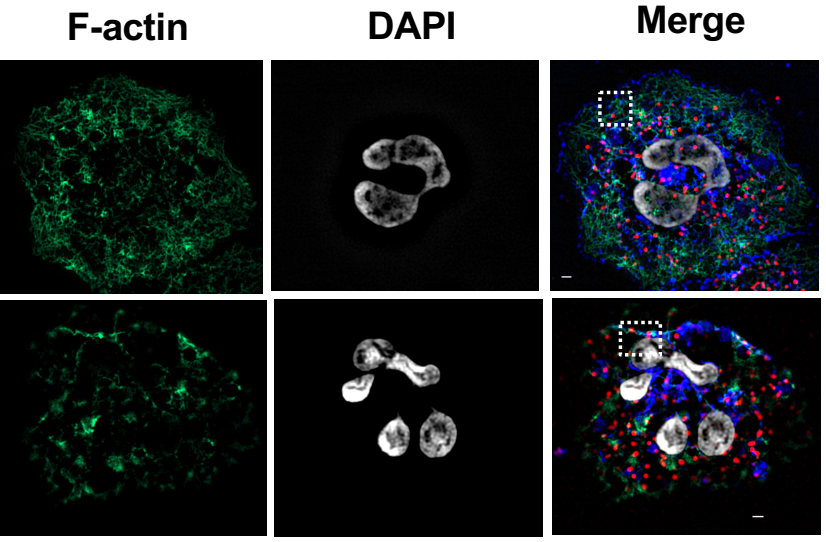

Scale bar $1 \mu \mathrm{m}$

Zoom
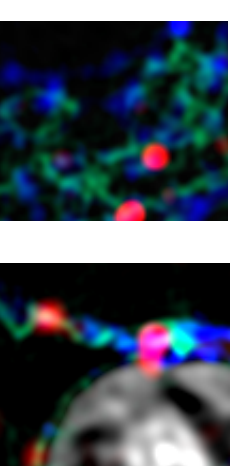

G

[- $\mathrm{rPMN} \square$ CCR5 cPMN $\square \mathrm{CCR}^{+}$cPMN

H

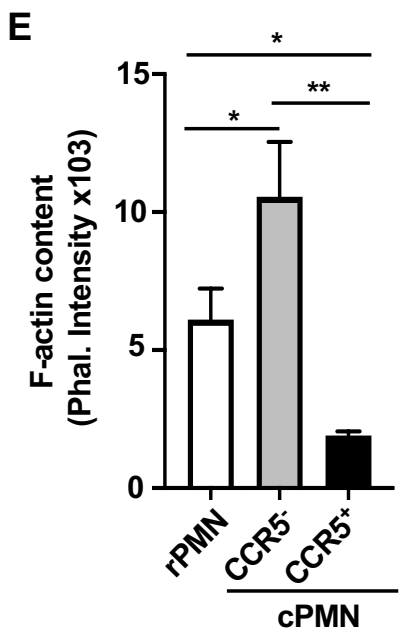

$\mathbf{F}$

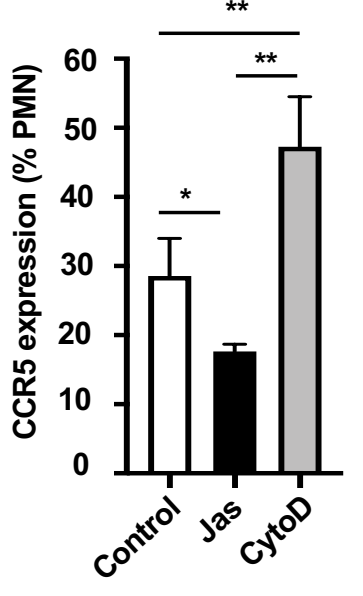

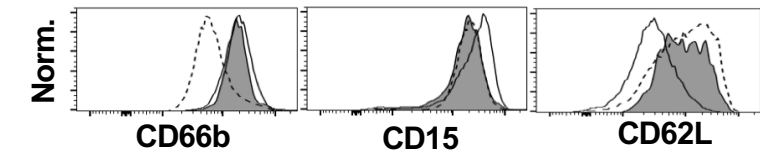

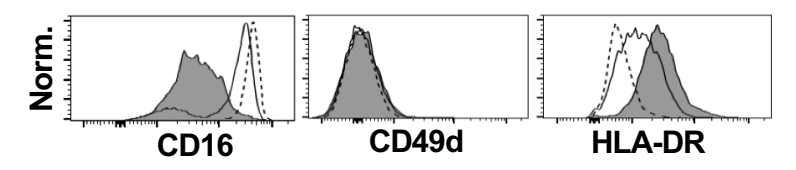

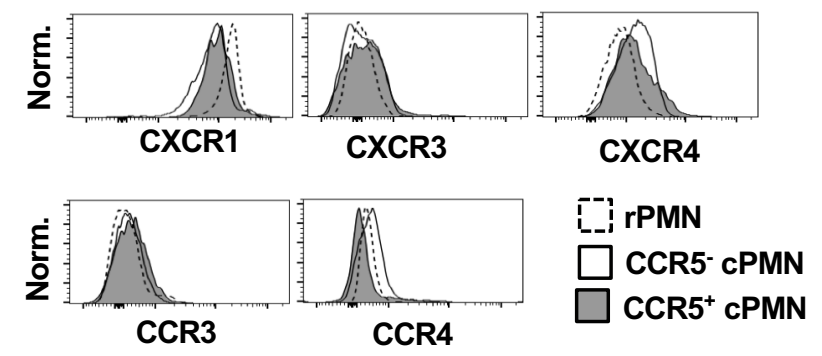


Figure 3

A

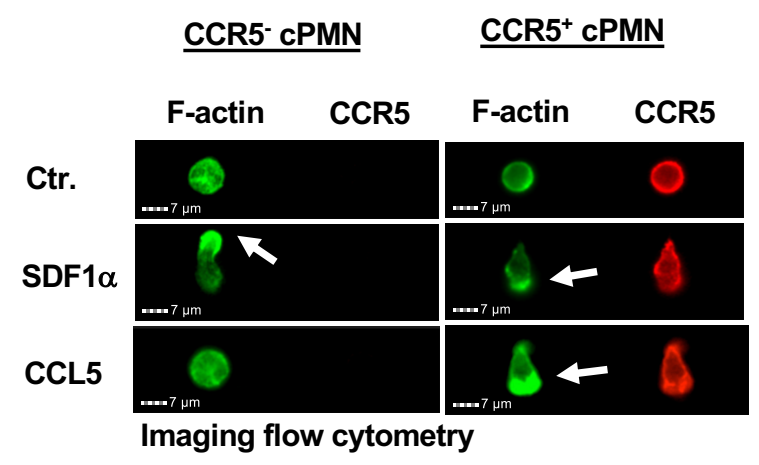

C

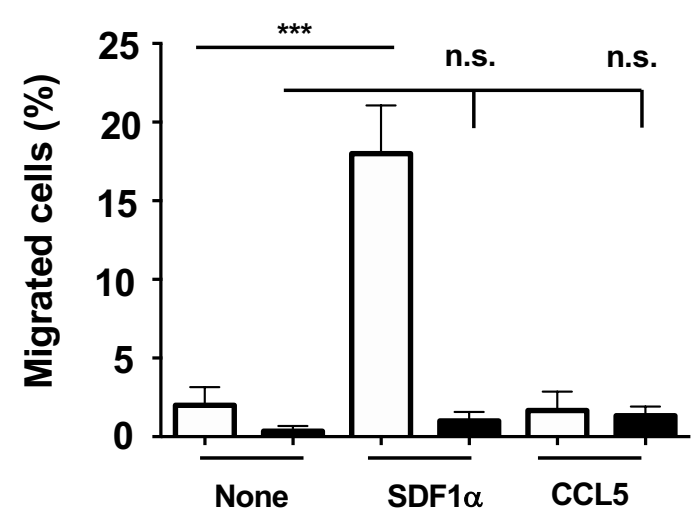

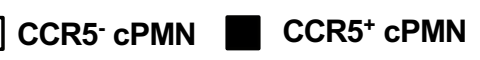

B

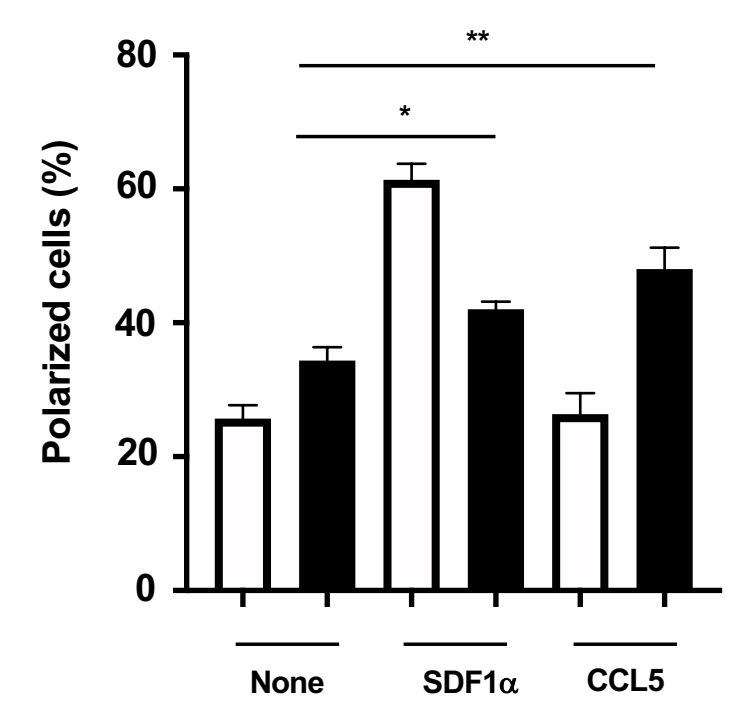

$\mathrm{CCR}^{-} \mathrm{cPMN} \square \mathrm{CCR}^{+} \mathrm{cPMN}$
rPMN

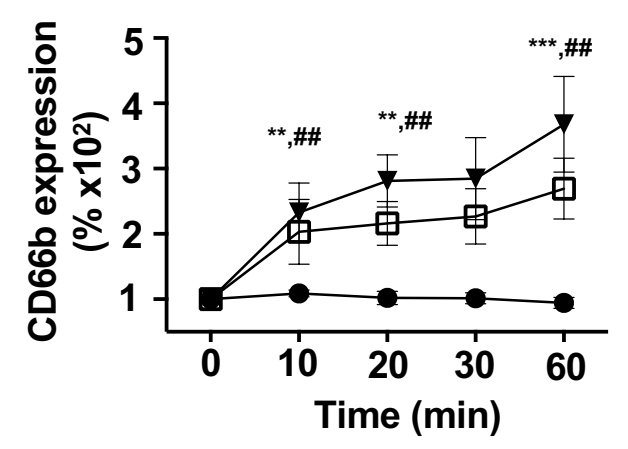

CCR5- CPMN

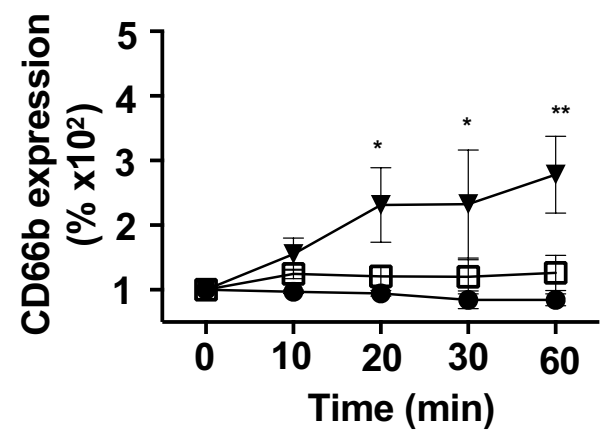

$\mathrm{CCR}^{+}$CPMN

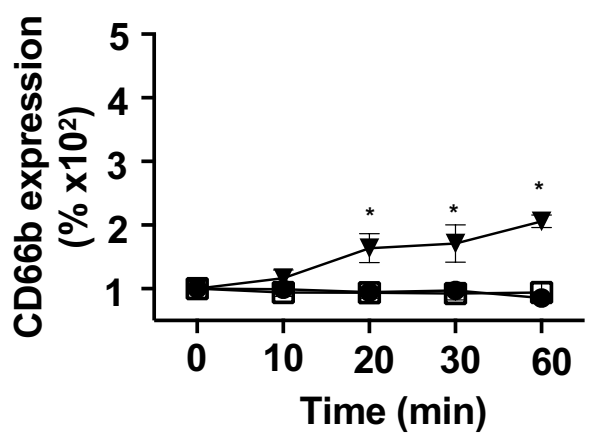

Unst. $\square$ fMLP $\nabla$ lonomycin

E

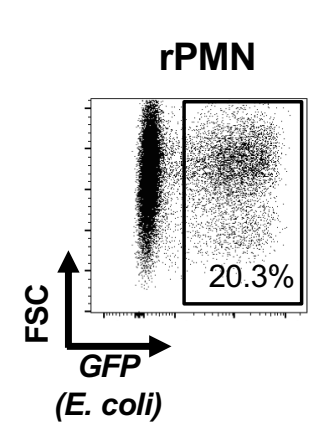

F

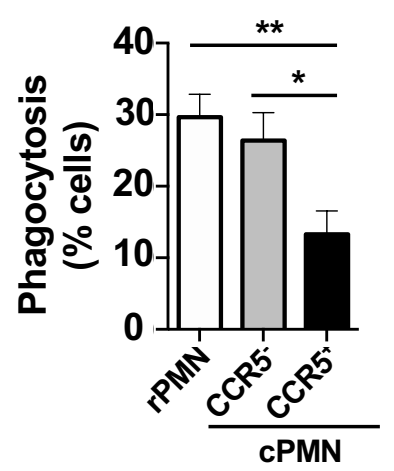


Figure 4

A

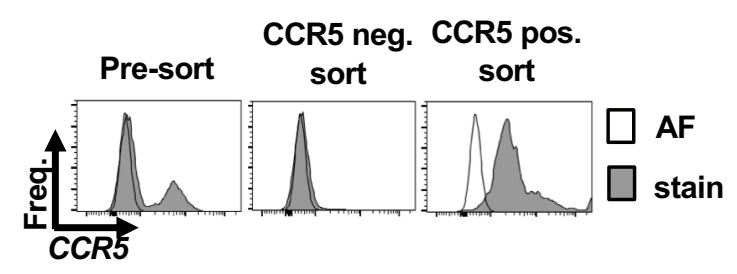

B

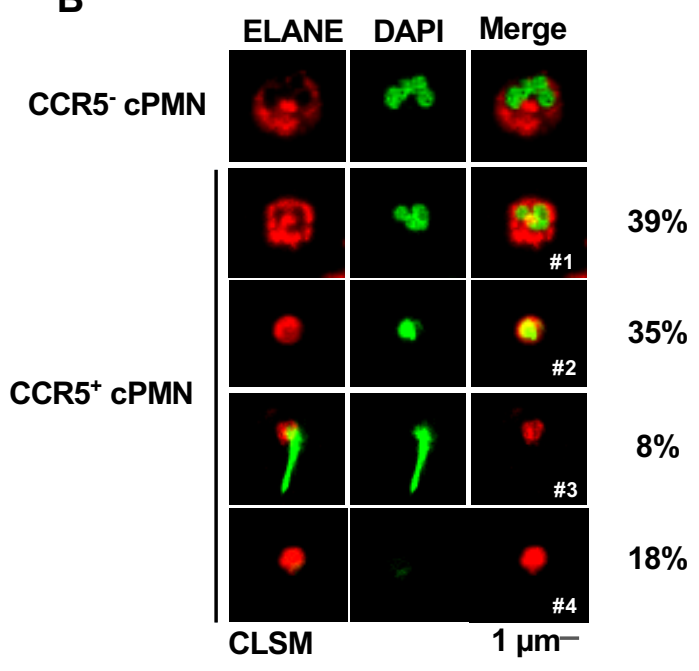

C

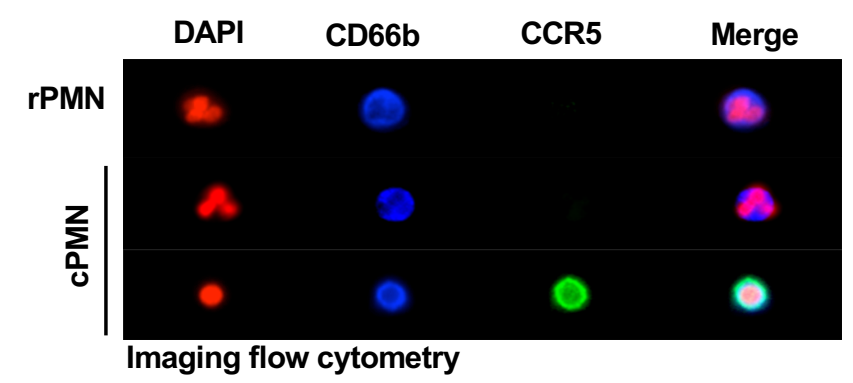

D

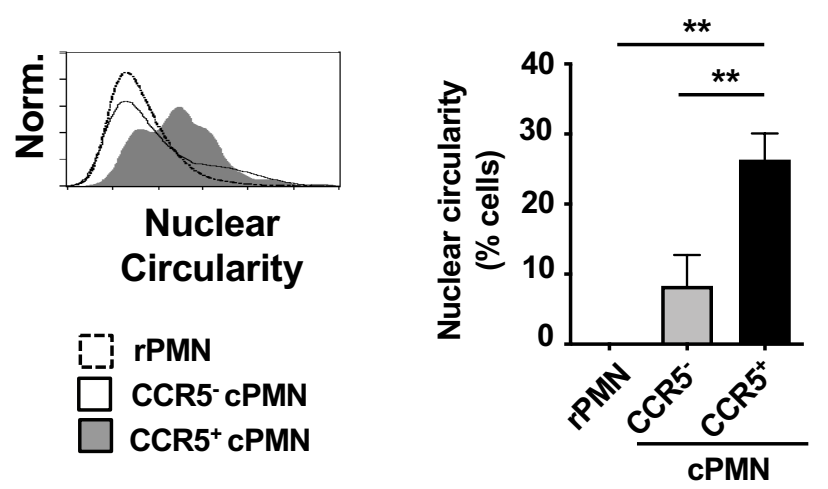

E
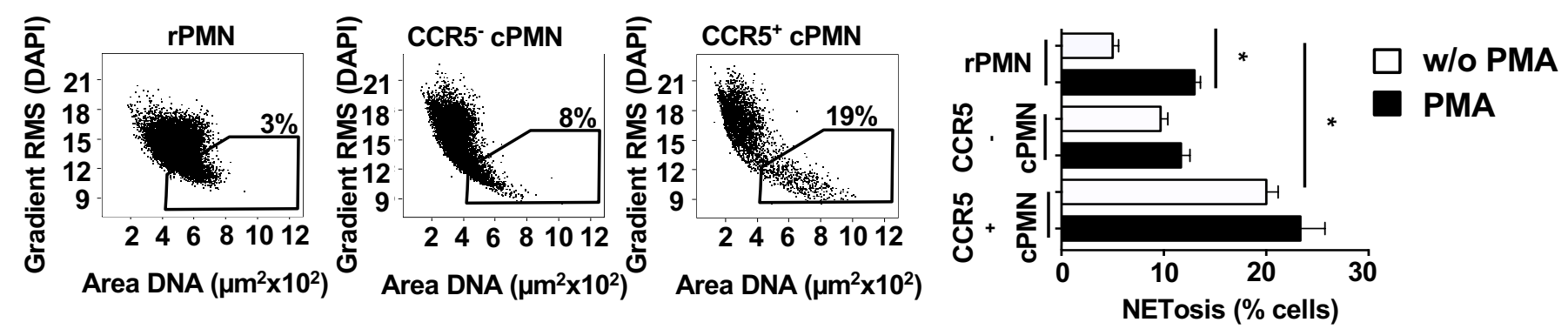

F

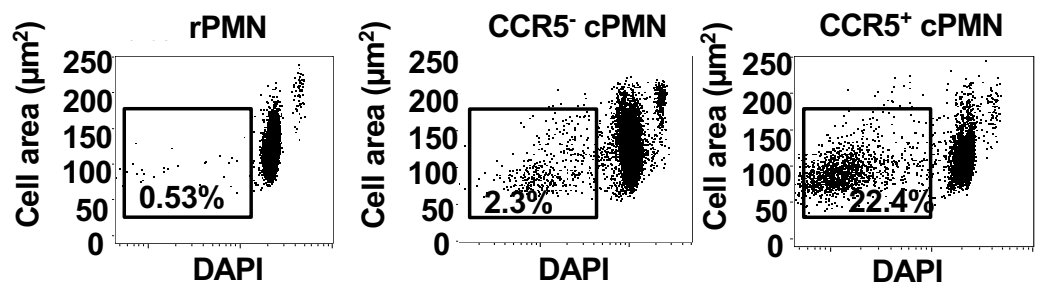

G
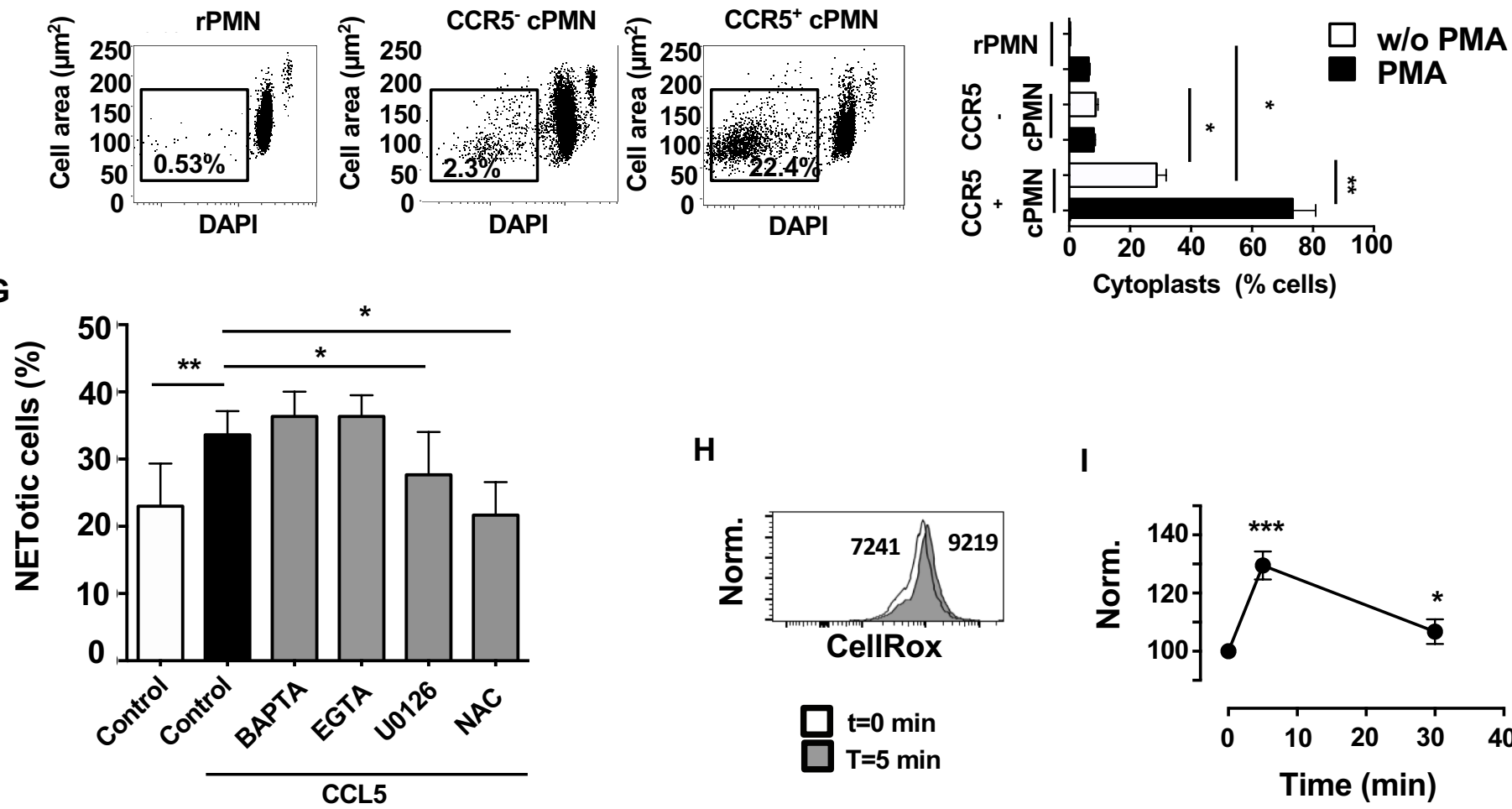

H

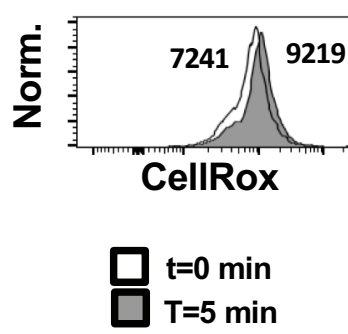

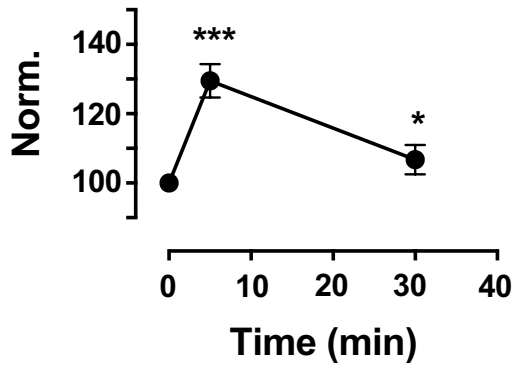


Figure 5

A

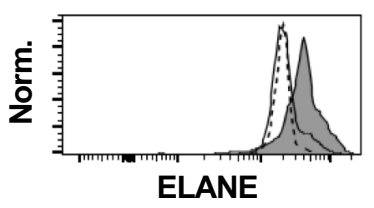

II rPMN

CCR5-CPMN

$\square \mathrm{CCR}^{+} \mathrm{CPMN}$

D

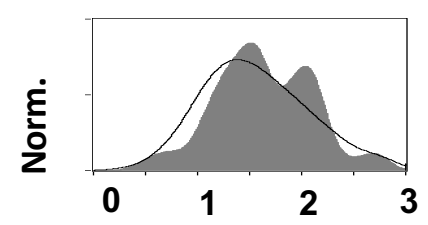

Similarity ELANE/DAPI

CCR5- CPMN $\square$ CCR5 $^{+}$CPMN
B

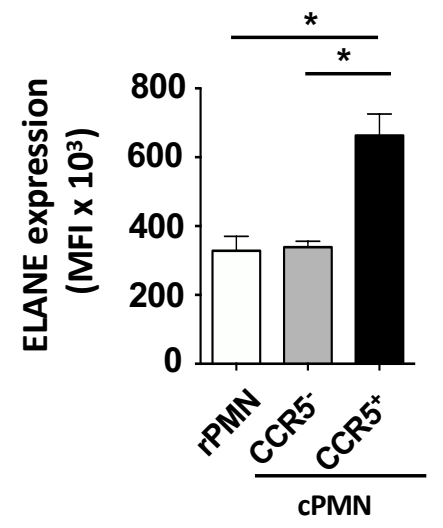

E

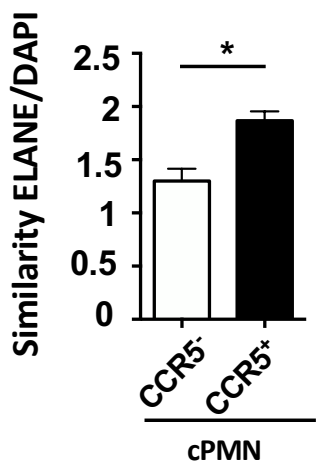

C

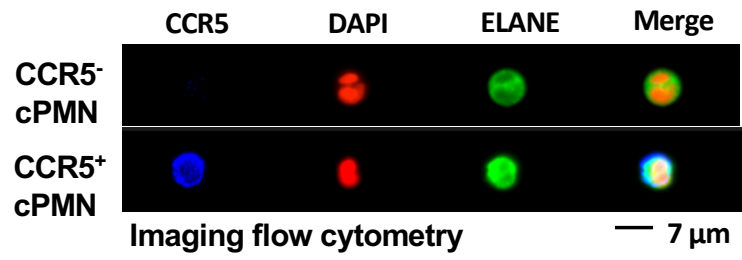


Figure 6

A
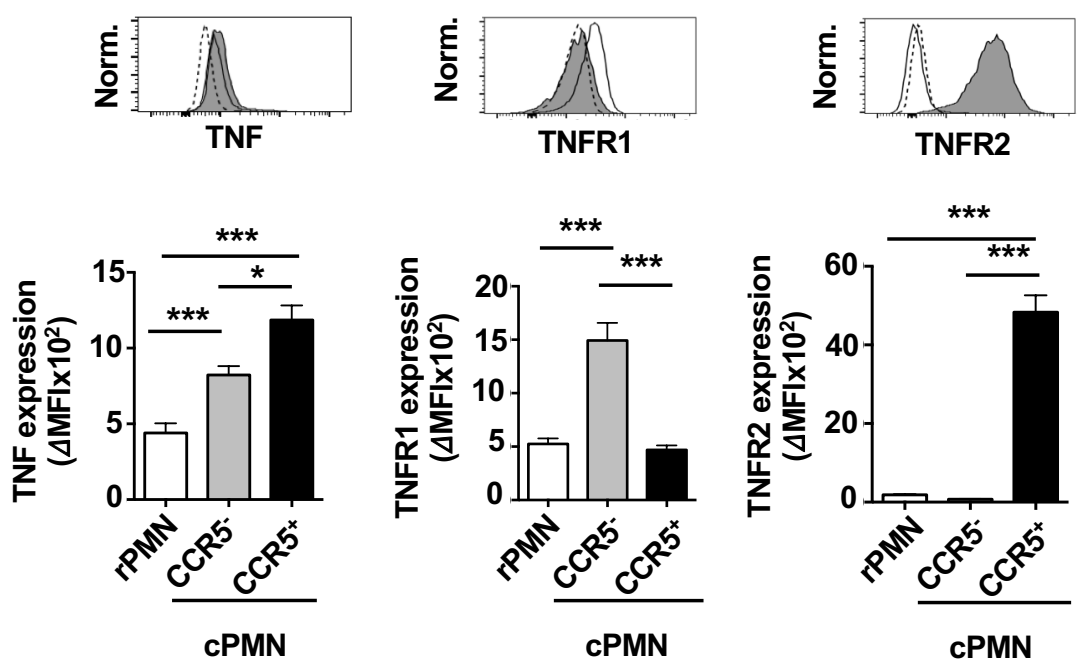

$\square$ rPMN $\square$ CCR5 CPMN $\square$ CCR5 $^{+}$CPMN

C

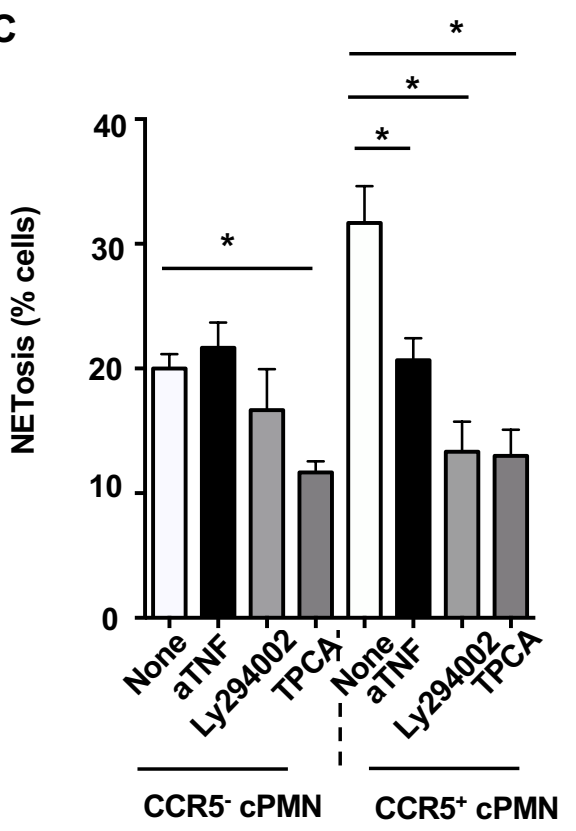

D

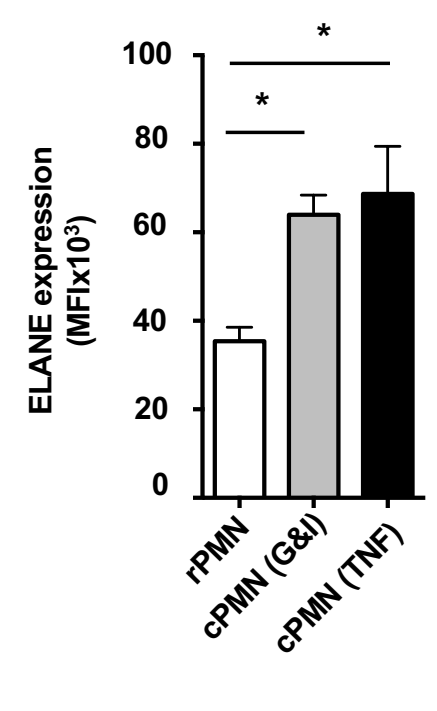

B

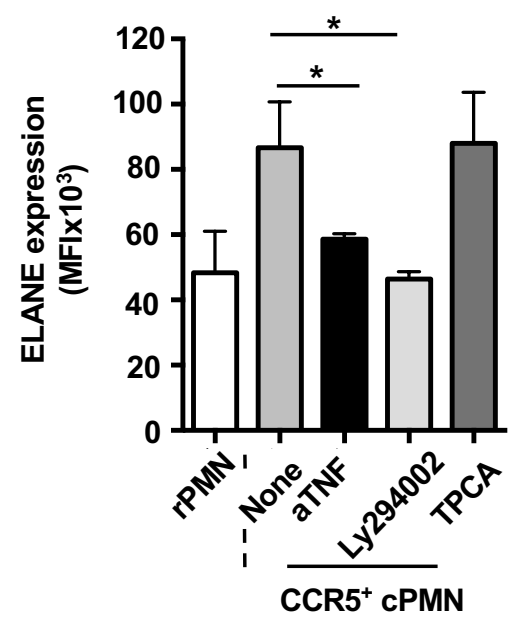

E
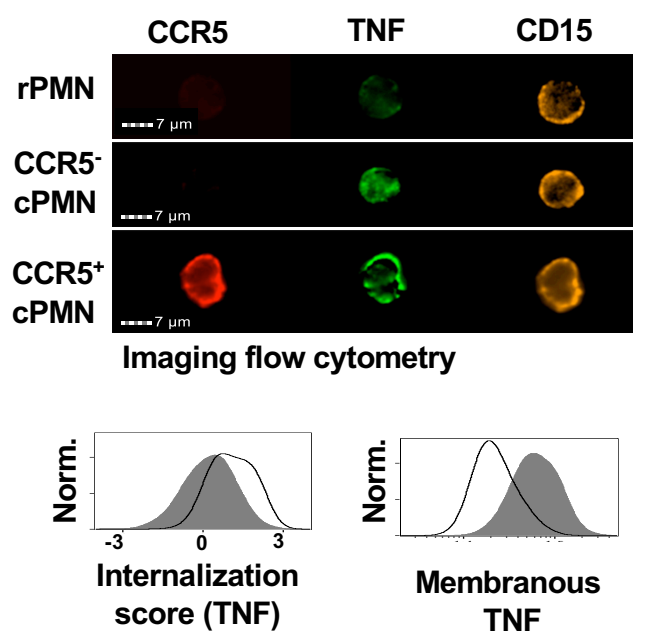

CCR5- CPMN

$\mathrm{CCR}^{+}$CPMN
F

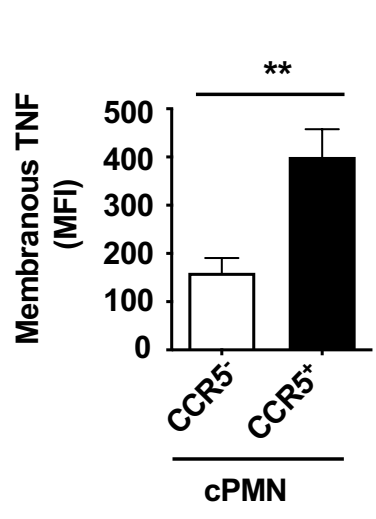

G

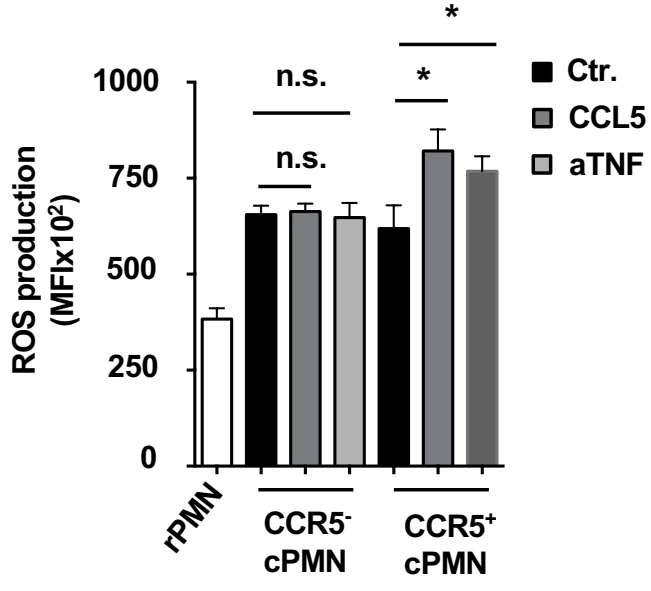

H

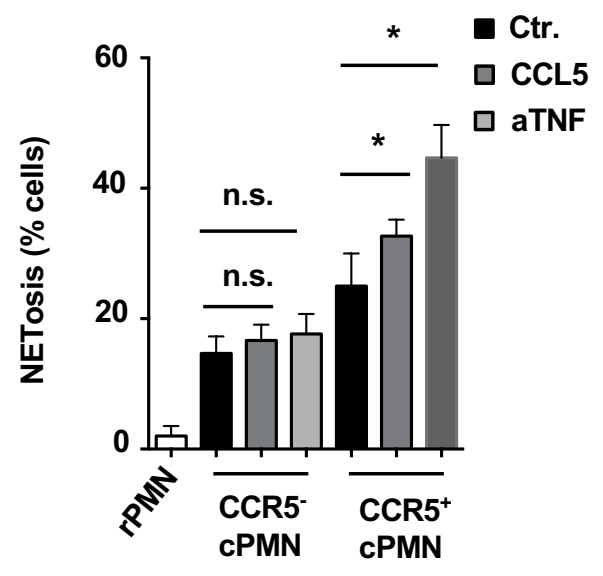


Figure 7

A

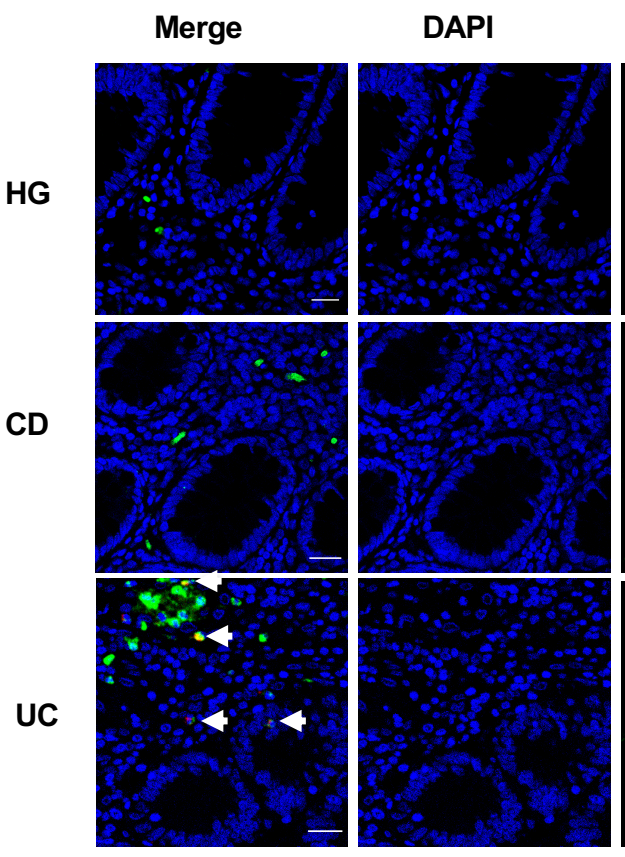

Confocal microscopy
Azurocidin
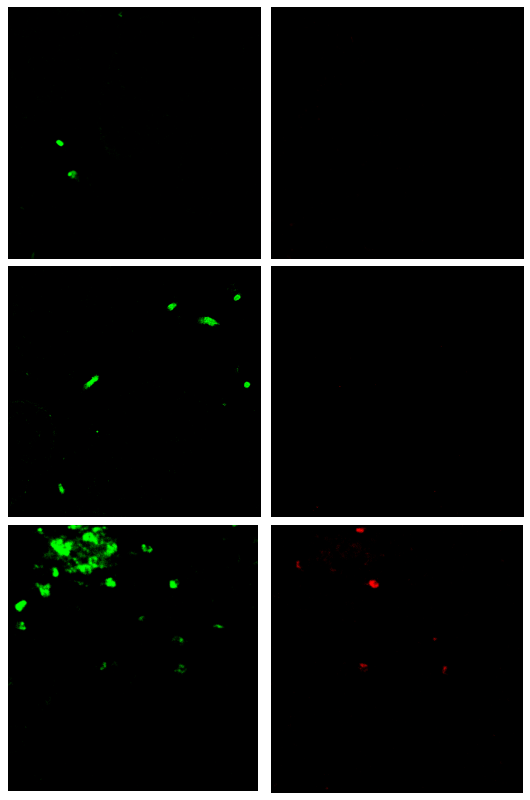

$25 \mu \mathrm{m}$

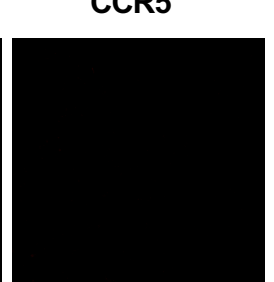

D

C

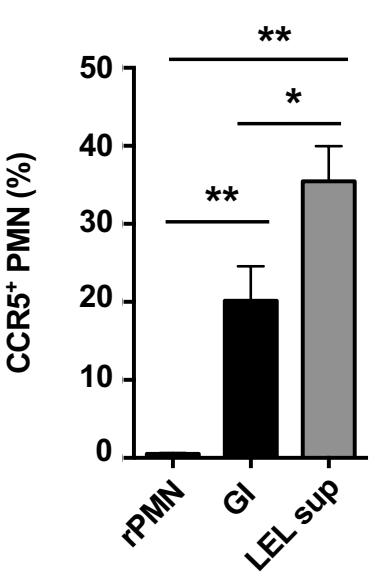

F

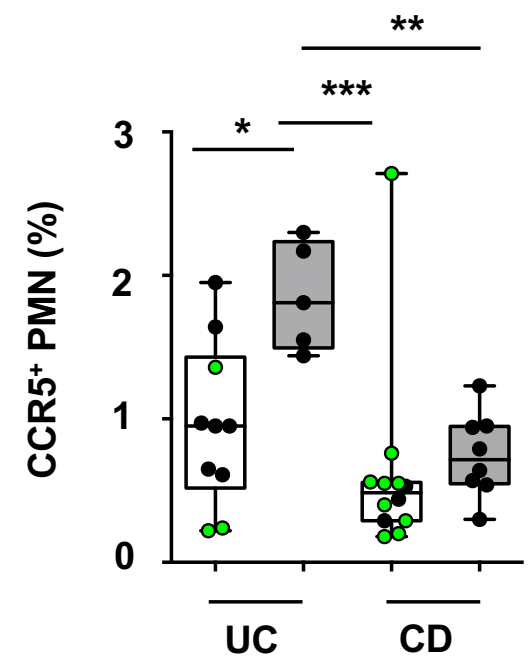

B

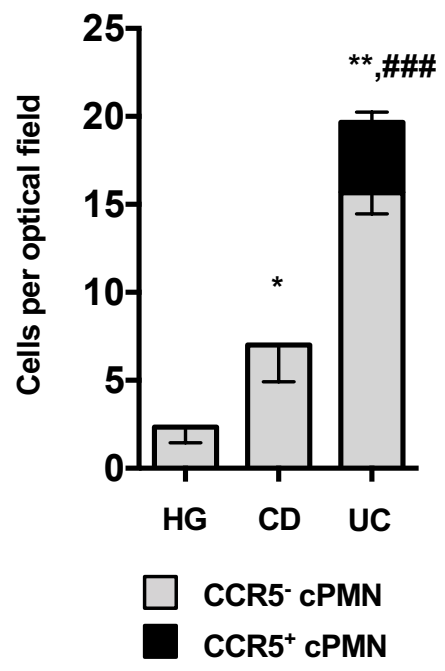

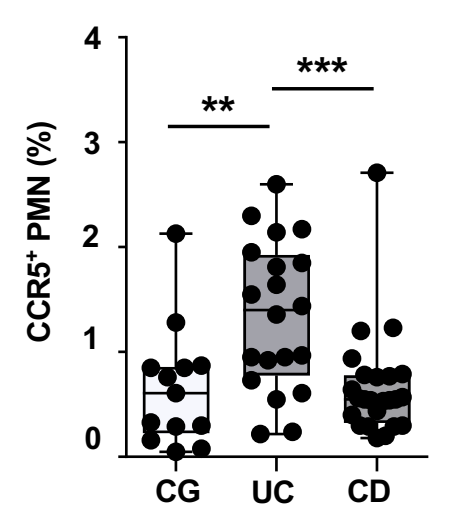

\begin{tabular}{|c|c|c|c|}
\hline & Parameter & $\mathbf{R}^{\mathbf{2}}$ & $\mathbf{p}$ \\
\hline \multirow{3}{*}{ CD } & $\mathrm{CRP}$ & 0.01158 & 0.6337 \\
\cline { 2 - 4 } & $\mathrm{HBI}$ & 0.01579 & 0.5874 \\
\cline { 2 - 4 } & fecal calprotectin & 0.00012 & 0.9616 \\
\hline \multirow{3}{*}{ UC } & CRP & 0.04603 & 0.4249 \\
\cline { 2 - 4 } & SCCAl & 0.06371 & 0.3840 \\
\cline { 2 - 4 } & fecal calprotectin & 0.04593 & 0.4619 \\
\hline
\end{tabular}

$\square$ O No anti-TNF therapy or

O successful anti-TNF therapy

Anti-TNF therapy failure

E 
Figure 8

A

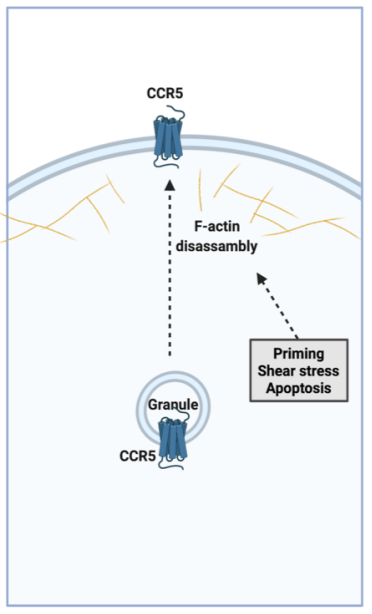

C

Anti-TNF during PMN diversification

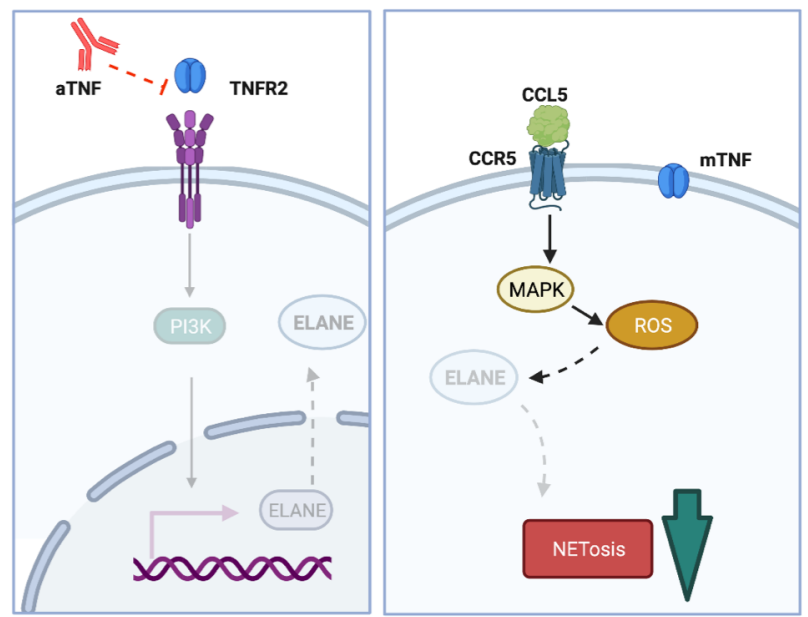

D

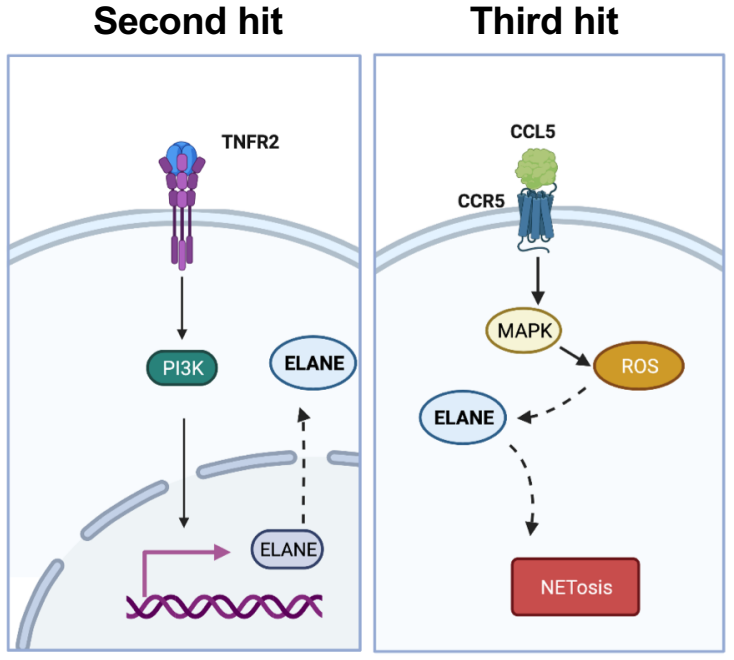

D

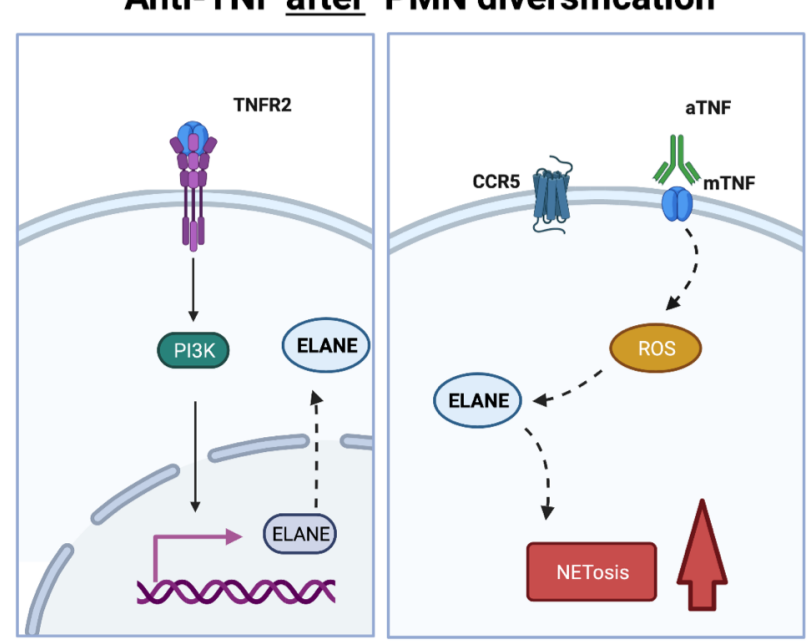

